\title{
Abstracts from
}

\section{The Aerosol Society}

Drug Delivery to

the Lungs 26

\section{Edinburgh International Conference Centre Edinburgh, Scotland, UK December 9-11, 2015}




\section{Abstracts: Drug Delivery to the Lungs 26}

\section{FACTORS AFFECTING DPI DEVELOPMENT FROM BENCH TO BEDSIDE}

\author{
A.H. de Boer
}

Department of Pharmaceutical Technology and Biopharmacy, University of Groningen, Groningen 9713 AV, The Netherlands

\section{Summary}

The factors that influence product development depend, amongst other things, on the type of product, the economic situation, the state of the art technology and various social and cultural trends. In modern era, often not the specs and costs are the decisive factors for purchasing a product, but aspects like design and brand personality. One of the reasons for this is the abundance of products of the same type for the same purpose. In fact, this is the current situation for dry powder inhalers (DPIs) although the self-congruity concept seems more applicable to the doctors who prescribe the inhalers than to the patients who are using them. For the early DPIs from the 1960s and 1970s the driving factor for development was the necessity of having an alternative for cholofluorocarbon (CFC) containing metered dose inhalers (MDIs). An important factor affecting their design could have been the desire to improve pulmonary drug delivery compared to that from MDIs, but unfortunately this oportunity was missed. Instead, available technology like hard gelatin capsules and adhesive ('ordered') mixtures were used and lung deposition remained more or less the same as from MDIs (only approx. 10\% of the dose). After the capsule (and multi-dose reservoir) DPIs became more widely accepted and patents on inhaled drugs expired, consulting groups and generic manufacturers became more and more involved in the emerging DPI market, purely on an economic basis. This does not mean that there have not been developments yet aiming at improvement of the therapy with DPIs and various future applications may give a new boost to using new technology for dry powder inhalation.

02. CHARACTERIZATION OF THE NANOSTRUCTURE, CRYSTALLINITY AND SPECIFIC SURFACE OF LACTOSE BASED INHALER POWDERS: SMALL-AND WIDE-ANGLE X-RAY SCATTERING (SWAXS) STUDIES $\frac{\text { Verena Wahl }^{1} \text {, Andreas Zimmer }}{2}$, Johannes Khinast ${ }^{1,3}$,

${ }^{1}$ Research Center Pharmaceutical Engineering, Inffeldgasse 13, Graz, 8010, Austria

${ }^{2}$ Karl Franzens University of Graz, Institute of Pharmaceutical Science, Universitätsplatz 1, Graz, 8010, Austria

${ }^{3}$ Institute for Process and Particle Engineering, Graz University of Technology, Inffeldgasse 13, Graz, 8010, Austria

${ }^{4}$ Bruker AXS, Angelo-Eustacchio Gasse, Graz, 8010, Austria

\section{Summary}

The aim of this work was to implement simultaneous the small- and wide- angle X-ray scattering (SWAXS) technique in the character- ization of dry powder inhaler (DPI) carriers and formulation. SWAXS data were used to identify changes in crystallinity and inner structure (specific surface) of diverse inhalable lactose micro-particles originating from particle surface manipulation process. During the wet surface processing of carrier material a high amount of fines were produced identified as a mixture of $\alpha$-lactose monohydrate and anhydrous lactose by a detailed analysis via SWAXS. Furthermore, to envisage the impact of environmental factors relevant to the processing, packaging and storage on the formulation attributes and performance selected lactose based commercial DPI formulations were exposed to accelerated storage conditions and microstructural evolution was investigated by SWAXS at regular time intervals. It was shown that during the storage of two commercial DPI products at different relative humidities, the specific surface of the formulation decreased significantly while no differences in the crystallographic pattern were noticeable. To prove the results, complementary techniques were implemented in the study (e.g. differential scanning calorimetry). Combined small- and wide- angle X-ray scattering seems to be a promising technique to identify changes in the particulate and the solid-states of DPI formulations and to predict physical stability.

\section{DEVELOPMENT OF MANNITOL-BASED COMPOSITE PARTICLES BY SPRAY DRYING: UNCOVERING THE CRITICAL PROCESS PARAMETERS}

\author{
Eunice Costa, Filipa M. Maia, Susana Campos, and Filipe Neves
}

Hovione FarmaCiencia, SA, Sete Casas, 2674-506 Loures, Portugal

Mannitol has been extensively explored in dry powder inhalation (DPI), namely i) as an alternative to lactose monohydrate in carrierbased formulations, being a non-reducing sugar alcohol, ii) for the treatment of cystic fibrosis as an airway hydrating agent and iii) for bronchial provocation testing. Moreover, mannitol holds great potential for engineering DPI composite particle formulations by spray drying (SD), since it is highly crystalline after the process, ensuring enhanced physical stability.

Although it is known that mannitol particle properties can be manipulated by adjusting SD parameters, few works can be found in the literature, especially on the field of respirable composite DPI formulations. For this reason, the current study reports the impact of SD parameters on mannitol particle size, morphology and solid state properties, aiming at optimal aerodynamic performance, using a design of experiments (DoE) approach. The best performing system was co-spray dried with nanoparticles of a model active pharmaceutical ingredient (API).

The aerodynamic performance of the mannitol particles were significantly impacted by the drying temperature, since particle morphology changed substantially within the explored range, due to differences in crystallization kinetics. The feed solution concentration, contrarily to expectation, was not a very significant parameter and the best performing system considered an intermediate temperature/feed concentration. Moreover, inclusion of the nanoparticles did not 
change significantly the deposition profile, regardless of the API relative concentration.

The illustrated development strategy allows a priori optimization of complex crystallizing composite particles, while balancing performance with process throughput. The resulting particles should be particularly suitable for dose-ranging studies, given its negligible dependence on the API load.

\section{MOVING FROM LOW SHEAR TO HIGH SHEAR BLENDING: A PREDICTABLE SCALE-UP OR A SOURCE OF UNCERTAINTY?}

Filipa M. Maia, Isabel S. Lopes, Maria Palha and Filipe Neves

Hovione FarmaCiencia, SA, Sete Casas, 2674-506 Loures, Portugal

Dry powder inhalation formulations include an excipient and an active pharmaceutical ingredient (API) which are present in different proportions and must be homogeneously blended. There are mainly two kinds of mixers available in the market to perform this operation: low-shear and high-shear mixers. The predominant mechanisms of both types of equipment are different and may impact the final mixture in terms of: (i) quality of mixing, (ii) aerodynamic performance and (iii) formulation stability. This work intends to compare the first two attributes stated above when using both types of blenders. For this purpose, six blends were produced in Turbula ${ }^{\circledR}$ (low-shear) and in Diosna $^{\circledR}$ (high-shear) units and were evaluated for blend homogeneity and aerodynamic performance.

The obtained results show that even though the blend uniformity was very similar for both processes, the quality of mixing given by the mixing index (MI) is higher for blends processed in the high-shear unit. Additionally, increasing mixing time and velocity did not have an impact on the quality of mixing, within the ranges explored in this work. Regarding aerodynamic performance, a decrease of the emitted dose (ED) and the fine particle dose (FPD) was observed for highshear blends. Additional mixing steps on the high-shear blends proved to have an impact on the aerodynamic performance, since the optimum mixing time appears to have been achieved in the first additional step, after which a re-segregation may have occurred, with a detrimental impact on the in-vitro deposition profile. When moving from low-shear into high-shear blending, the observations drawn from this work should, therefore, be taken into consideration.

\section{INFLUENCE OF FINES ON COMMERCIAL LACTOSE CARRIERS AND THEIR DRY POWDER INHALATION PERFORMANCE}

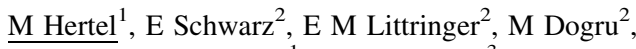
R Scherließ ${ }^{1}$, and $\mathrm{H} \mathrm{Steckel}^{3}$

${ }^{1}$ Department of Pharmaceutics and Biopharmaceutics, Kiel University, Grasweg 9a, 24118 Kiel, Germany

${ }^{2}$ Molkerei Meggle Wasserburg GmbH \& Co. KG, Megglestr 6 - 12, 83512 Wasserburg am Inn, Germany

${ }^{3}$ Deva Holding AS, Istanbul, Turkey

Many efforts have been made in the past to explain the beneficial effect of fines but the exact mechanisms how these fine lactose particles alter the performance of dry powder inhaler (DPI) formulations has remained unclear. In this study the influence of fines added to commercially available lactose carriers is investigated. For this purpose, two different-sized carriers were blended with different concentrations of fines and the model drug budesonide in different blending orders. All blends were prepared with a high shear mixer, which would make an upscaling possible, at different rotational speeds and mixing times. Afterwards the blends were tested with an inhaler device $\left(\right.$ Novolizer $\left.^{\circledR}\right)$ by using the Next Generation Pharmaceutical Impactor (NGI). The fine particle fraction (FPF), the fine particle dose (FPD) and the mass median aerodynamic diameter (MMAD) were then compared with the factors rotation speed, blending time, blending order and the amount of fines. It could be shown that all factors have an impact on the FPF and FPD. As an explanation all common postulated theories come into consideration. With the help of blending order results and SEM pictures the saturation of active sites, the function of the fines as a buffer during blending and the formation of drug-fines agglomerate could be proven. Overall, the great benefit of fines for commercial lactose carrier and their DPI performance could be shown in this work as well as the importance of a better understanding of the exact mechanism of their acting, in particular for the blending process.

\section{CHARACTERISATION OF PARTICLE ENGINEERED MANNITOL AS ALTERNATIVE CARRIERS IN DRY POWDER INHALATION FORMULATIONS}

\author{
$\underline{\text { N Rhein }}^{1}$, G Birk ${ }^{2}$, and R Scherließ ${ }^{1}$ \\ ${ }^{1}$ Department of Pharmaceutics and Biopharmaceutics, \\ Kiel University, Grasweg 9a, 24118 Kiel, Germany \\ ${ }^{2}$ Merck KGaA, Frankfurter Straße 250, 64293 Darmstadt, Germany
}

Lactose is the typical carrier particle in carrier based powder blends for inhalation. Particle engineered Mannitol A and B are tested as potential alternative to the established carrier lactose in dry powder inhaler (DPI) formulations. Particle engineered Mannitol is a wellknown product for direct compression in blends with low content of active pharmaceutical ingredients (API). Particle engineered Mannitol $\mathrm{A}$ and $\mathrm{B}$ are characterised with respect to their physico-chemical properties (particle size distribution, density, crystallinity, hygroscopicity). They are also as carrier material in interactive blends for DPIs and are investigated with respect to their suitability for inhalation (homogeneity and fine particle dose). It is shown that particle engineered Mannitol is a solid, crystalline and non-hygroscopic powder which shows a constant dosing from reservoir-based inhalers. The physical stability is an important factor for storage stability. Blends with an API content of $1.5 \%$ exhibit a low relative standard deviation under $3 \%$ and an FPF of $24.68 \%$ indicating that particle engineered Mannitol could be a good alternative carrier in DPI blends.

\section{SPACE CHAMBER DEVICES IN VITRO PERFORMANCE EVALUATION AT CONSTANT FLOW}

\author{
R F Oliveira $^{1}$, S F C F Teixeira ${ }^{2}$, \\ H M Cabral-Marques $^{3}$, and J C F Teixeira ${ }^{1}$ \\ ${ }^{1}$ CT2M R\&D Centre, University of Minho, 4800-058 Guimarães, \\ Portugal \\ ${ }^{2}$ ALGORITMI R\&D Centre, University of Minho, 4800-058 \\ Guimarães, Portugal \\ ${ }^{3}$ iMed. ULisboa R\&D Centre, Universidade de Lisboa, 1649-003 \\ Lisboa, Portugal
}

Background: The use of spacers is advisable for asthma treatment in younger patients $(<5$ years). This work aims to evaluate such devices in terms of performance when compared against the use of pressurized Metered-Dose Inhaler (pMDI) solo. 
Methods: For that purpose an experimental in vitro assessment of four valved holding chambers (VHC) and one tubular spacer was made using a Multi-Stage Liquid Impinger at $60 \mathrm{~L} / \mathrm{min}$. The add-on devices were tested with Ventolin HFA-134a (salbutamol sulphate, as API), and the drug deposited in the setup was recovered with $\mathrm{NaOH}$ $0.01 \mathrm{M}$. Solutions concentrations were estimated by UV-Vis spectrophotometry at $244 \mathrm{~nm}$.

Results: Results showed that the highest VHC mass deposition was found in the Volumatic ${ }^{\circledR}$, while the valveless tubular spacer (i.e. Compact Space Chamber Spacer ${ }^{\circledR}$ ) has the lowest deposition. Add-on devices throat deposition was found to be lower than the pMDI solo $\left(\approx 45 \mu \mathrm{g}\right.$ ). Only between the Compact Space Chamber ${ }^{\circledR}$ and the Space Chamber ${ }^{\circledR}$ were not found statistically significant differences in throat deposition $(\mathrm{p}>0.05)$. The mass median aerodynamic diameter (MMAD) is lowest for the Volumatic ${ }^{\circledR}$ and highest $(2.3 \pm 0.1 \mu \mathrm{m})$ for the valveless tubular spacer $(3.2 \pm 0.3 \mu \mathrm{m})$, with statistical differences between the add-on devices $(\mathrm{p}<0.001)$. The MMAD for VHCs with leaflets valve type showed no significant differences $(p>0.05)$. Fine particle mass (FPM) between the add-on devices showed no differences $(\mathrm{p}>0.05)$. The highest FPM is provided by Volumatic ${ }^{\circledR}$ $(36.5 \pm 2.4 \mu \mathrm{g})$ and the lowest Compact Space Chamber Anti-static ${ }^{\circledR}$ $(32.9 \pm 3.2 \mu \mathrm{g})$. The pMDI solo emits lower FPM $(29.3 \pm 3.4 \mu \mathrm{g})$. The calculation of the coarse/fine/extra-fine particle fractions and coarse/fine/extra-fine ratios showed that all the add-on devices have similar performance results for all the calculated metrics, being within the reference values.

Conclusions: Add-on devices provide the reduction of the coarse fraction of the pMDI plume and, subsequently, the reduction of the throat deposition. The existence (or not) of a valve, even the type of valve, has influence in the mass deposited inside the VHC and in the throat.

\section{APPLYING QBD PRINCIPLES IN EARLY DPI DEVELOPMENT: DESIGNING FOR ROBUSTNESS THROUGH DESIGN SPACE EXPLORATION}

João V. Fernandes, Isabel S. Lopes, and Filipe Neves

Hovione FarmaCiencia SA, Sete Casas, 2674-506 Loures, Portugal

A major benefit of performing Design Space exploration as early as possible during Dry Powder Inhaler (DPI) development is the identification of optimal design parameters as well as the quantification of the robustness of such optimal design points. Design Space exploration is a cornerstone of Quality by Design $(\mathrm{QbD})$ based approaches to pharmaceutical product development and its application to inhalation device development has been proposed.

Within the context of the development of an enhanced version of the currently marketed TwinCaps ${ }^{\circledR}$ DPI with an enlarged powder cavity targeting effective high dosage drug delivery to the lungs, the current work reports an exploration of the Design Space considering the interactions of device, particle engineering and formulation design variables on key inhalation drug product performance attributes, such as Emitted Mass (EM) and Fine Particle Fraction $\left(\mathrm{FPF}_{5 \mu \mathrm{m} / \mathrm{EM}}\right)$. For that purpose, an experimental characterization of the Design Space defined by the i) device powder cavity outlet blockage $\sigma$, defined as the ratio between obstruction and total channel area (design parameter), ii) model drug particle size and iii) formulation fill weight, was performed using rapid prototyping technologies together with fast screening analytical methods.

Results from the current Design Space exploration revealed that the selection of a device powder outlet blockage between 0.7 and 0.85 delivers a robust EM and $\mathrm{FPF}_{5 \mu \mathrm{m} / \mathrm{EM}}$ performance across the interval of particle size and formulation fill weight values evaluated.

\section{INVESTIGATION OF DRY POWDER INHALATION AEROSOLISATION PERFORMANCE AT DIFFERENT FLOW RATES FROM A CONVENTIONAL CAPSULE-BASED INHALER DEVICE}

\author{
$\underline{\text { I Y Saleem }}^{1}$, R KR Rajoli ${ }^{1}$, and F Diez ${ }^{2}$
}

\author{
${ }^{1}$ School of Pharmacy and Biomolecular Sciences, Liverpool \\ John Moores University, Liverpool, L3 3AF, UK \\ ${ }^{2}$ Qualicaps Europe, S.A.U., Calle de la Granja 49, 28108 \\ Alcobendas (Madrid), Spain
}

Background: Generally capsule-based dry powder inhalers (DPIs) are required to be used at a flow rate of $60 \mathrm{~L} / \mathrm{min}$ to initiate removal and deaggregation of the drug from carrier for effective pulmonary deposition. This may not be appropriate for children or those with lung diseases. In addition, capsule-based DPI are intended for treatment over a 4-week period and storage conditions can influence the aerosolisation drug deposition, reproducibility of inhalation dose and treatment outcome.

Methods: Inhalation grade lactose was blended with micronized salbutamol $(50: 1 \mathrm{w} / \mathrm{w})$ and filled $(20 \pm 1 \mathrm{mg})$ in to size 3 hypromellose capsules stored at $22^{\circ} \mathrm{C} 40 \% \mathrm{RH}$ for 4 weeks. Samples were tested using a 2-pin inhaler and aerosolised through a next generation impactor at a flow rate of $30 \mathrm{~L} / \mathrm{min}$ (actuated for $8 \mathrm{~s}$ ) and $60 \mathrm{~L} / \mathrm{min}$ (actuated for 4s) at weekly intervals for 4 weeks. Deposition of drug in the capsule, device and emitted dose (ED), fine particle dose (FPD), \% fine particle fraction (FPF) and mass aerodynamic diameter (MMAD) were calculated.

Results: Powder retention within capsules was higher at $60 \mathrm{~L} / \mathrm{min}$ whereas it was higher in the device at $30 \mathrm{~L} / \mathrm{min}$ ( $\mathrm{p}<0.05 /$ Tukey). The $\mathrm{ED}, \mathrm{FPD}, \mathrm{FPF}$ was significantly greater at $60 \mathrm{~L} / \mathrm{min}$ compared to 30 $\mathrm{L} / \mathrm{min}$ at each time point $(\mathrm{p}<0.05 /$ Tukey $)$. However, there was very little significant difference comparing each flow rate over time.

Conclusion: Differences in results between the air flow rates at each weekly time points highlights the important relationship between inhalation, therapeutic dose, lung deposition and potentially therapeutic outcome.

\section{EFFECT OF THE BREATH-ACTUATED MECHANISM ON THE DISPERSION PERFORMANCE OF THE NEXTHALER ${ }^{\circledR}$}

\author{
F Mason, A Tweedie, and D A Lewis
}

Chiesi Limited, Chippenham, Wiltshire, SN14 OAB, UK

\section{Summary}

Background: The innovative breath-actuated mechanism (BAM) within the NEXThaler ${ }^{\circledR}$ controls the dose release in response to pressure drop. The effect of the BAM on the dispersion performance from NEXThaler will be evaluated using in-vivo inhalation profiles.

Methods: The dispersion performance of a $100 \mu \mathrm{g} / \mathrm{dose}$ of Beclometasone Dipropionate (BDP) formulation was assessed, using invivo asthmatic inhalation profiles specific to the NEXThaler ${ }^{[1]}$. The three profiles differed notably from each other with peak inhalation flow rates of 45, 56 and $100 \mathrm{~L} \mathrm{~min}^{-1}$.

Results: There was a noticeable reduction in dispersion performance when the BAM was removed from the NEXThaler device; $51 \% \pm 3 \%$ compared to $37 \% \pm 6 \%$ on average across all three profiles. Dose evacuation profiles demonstrate that without the presence of a BAM the dose is released at the same time, near the start of the inhalation, regardless of the inhalation profile $(0.27 \pm 0.01 \mathrm{~s})$. The NEXThaler Control BAM releases the dose only when a pressure drop 
of approximately $2 \mathrm{kPa}$ has been reached. Removal of the BAM causes the dose to be released into a slower airflow velocity, 9-11 $\mathrm{L} \mathrm{min}^{-1}$ for the No-BAM device variant and $36-37 \mathrm{~L} \mathrm{~min}^{-1}$ for the NEXThaler Control. This may mean that larger carrier particles are less likely to impact within the device and could reduce the mass of fine active pharmaceutical ingredient (API) detaching from the carrier particles.

Conclusion: Dispersion performance can be improved by moderating the release point of the dose; this is exemplified by the NEXThaler device which includes a BAM.

\section{EXTENDED COMPARISON BETWEEN GRAVIMETRIC IMPACTORS FOR THE EVALUATION OF COMPOSITE AND HIGH DOSAGE COMPOUNDS FOR INHALATION}

\author{
Isabel S. Lopes, Maria Palha, Sofia Silva, and Filipe Neves
}

Hovione FarmaCiencia, SA, Sete Casas, 2674-506 Loures, Portugal

The development of dry powder inhalers (DPIs) and/or inhalation formulations is a laborious process which involves the determination of aerodynamic particle size distributions (aPSD), using full resolution impactors (i.e. Andersen Cascade Impactor (ACI) and the Next Generation Impactor (NGI)). Although developed to improve flexibility and precision, there is little information in the open literature regarding the benchmarking of the NGI with its predecessor, in their gravimetric form. Therefore, this work intends to compare both equipment and assess operational limits where the two techniques show good correlation. Additionally, characterization was also conducted with an abbreviated version of the NGI, the Fast Screening Impactor (FSI), to compare the results obtained via the three techniques (NGI, ACI, FSI) and, in this way, assess a potential full interchangeability.

The obtained results show that good correlations were found between the two full resolution impactors (NGI, ACI) when using fill weights of $20 \mathrm{mg}$. On the other hand, high fill weights (of $80 \mathrm{mg}$ ) were found to have a detrimental effect on the correlation, mostly due to an overload of the NGI apparatus. When comparing the FSI results with the corresponding full resolution results, a good correlation was found, in general; however, some loss of accuracy was noticeable, which translated into a reduced discriminative power, if in the presence of formulations of similar performance. Finally, all obtained results suggest that the use of gravimetric impactors are well suited for evaluating powders with a highly cohesive and adhesive nature, like the ones considered during the current work.

\section{UNDERSTANDING THE IN VIVO IMPACT OF CARRIER-DRUG INTER-PARTICLE FORCES VIA ENGINEERED SURFACE MODIFICATIONS: AN IN SILICO INVESTIGATION}

\author{
A Mercuri $^{1}, \mathrm{~S} \mathrm{Wu}^{1}$, S Zellnitz ${ }^{1}, \mathrm{~S}$ Salar-Behzadi ${ }^{1}$, \\ M Bresciani ${ }^{1}$, K Khinast $^{1}$, and E Fröhlich ${ }^{2}$ \\ ${ }^{1}$ Research Center Pharmaceutical Engineering GmbH, \\ Graz, 8010, Austria \\ ${ }^{2}$ Center for Medical Research, Medical University of Graz, \\ Graz, 8010, Austria
}

Engineered modifications of carrier surfaces are used to control the inter-particle forces occurring between the carrier and the drug. In this way, tailoring of these forces enables the quantity of drug that can be delivered to the lungs to be modulated. In this work the impact of engineered modification of carrier-drug surface forces was investigated using glass beads as model carrier particles. Untreated and treated glass beads mixed with salbutamol sulphate were characterised in vitro for their aerodynamic performance. The fine particle fraction (FPF) for treated beads was 1.36 times higher than that for untreated beads. In vivo pharmacokinetic prediction of the surface modifications was investigated after using a validated in silico pharmacokinetic (PK) model. The simulation showed that $\mathrm{C}_{\max }$ for salbutamol released from surface engineered beads was 1.20 fold that of untreated beads. Both in vitro and in silico data showed that increasing the surface roughness of the carrier increased the fine particle delivery performance of the formulation.

\section{IN-VITRO STABILITY AND DEGRADATION OF PLGA AND PGA-CO-PDL MICROPARTICLES FOR PULMONARY DRUG DELIVERY}

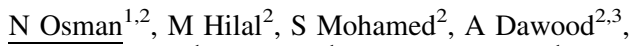 \\ K Ritchie $^{1}$, I Saleem ${ }^{1}$, and G Hutcheon ${ }^{1}$ \\ ${ }^{1}$ School of Pharmacy \& Biomolecular Sciences, Liverpool John \\ Moores University, Liverpool, L3 3AF, UK \\ ${ }^{2}$ Forensic Medicine \& Clinical Toxicology Dept., Sohag \\ University, Sohag, Egypt \\ ${ }^{3}$ Forensic Medicine \& Clinical Toxicology Dept., \\ Assuit University, Assuit, Egypt
}

Background: Aliphatic polyesters are widely used for drug delivery applications commonly poly (lactic-co-glycolic acid), PLGA. Their main drawbacks are the initial burst release and the resultant acidity at the site of drug action. A novel polyester; poly (glycerol adipate-co-w-pentadecalactone), PGA-co-PDL was synthesized and characterized to overcome these drawback. The study aim was to evaluate the stability and degradation of polymeric microparticles (MPs) prepared from PLGA and PGA-co-PDL under in-vitro pulmonary physiologic conditions as suspension in simulated lung fluid at $37^{\circ} \mathrm{C}$ under axial rotation $15 \mathrm{rpm} /$ minute.

Methods: MPs were formulated from PGA-co-PDL and PLGA by single emulsion method using Poly vinyl alcohol (PVA) as emulsifier. The MPs were characterized at specific time points for size, morphology and degradation features (by SEM), charge (By Zetasizer), $\mathrm{pH}$ (by pH meter) and molecular weight (By GPC).

Results: PGA-co-PDL MPs showed slower changes of size decrease, degradation changes and acidity over PLGA MPs. The Molecular weight changes over time confirmed that PGA-co-PDL MPs were slower degrading than PLGA ones. The charge was negative due to PVA and the trend over time indicated PGA-co-PDL MPs were more stable than PLGA ones

Conclusion: PGA-co-PDL MPs carriers had slower degradation and more stability than PLGA MPs under the stated experimental conditions. They are suitable carriers for sustained release formulations.

\section{FORMULATION OF BIOLOGICS FOR INHALED AND NASAL DELIVERY}

\author{
Mark Parry $^{1}$, Derek Solomon $^{1}$, Mark Hammond ${ }^{1}$, \\ David Ward $^{1}$, and Ashleigh Wake ${ }^{2}$ \\ ${ }^{1}$ Intertek Melbourn, Saxon Way, Melbourn, SG8 6DN, UK \\ ${ }^{2}$ Intertek Manchester, Hexagon Tower, Blackley, Manchester, \\ M9 $8 G Q, U K$
}

Biopharmaceuticals are a vital part of modern pharmaceutical development however they are susceptible to a range of aggregation and degradation pathways which make the manufacture, processing and delivery of these products more challenging than more typical small molecules, 
with the majority of biologics being delivered parenterally. Inhaled and nasal delivery of biologic products allows for a more convenient method of administering compounds systemically, and also allows direct targeting of the respiratory system. The use of many biologics for long term disease management provides opportunities for repurposing of existing products for inhaled and nasal delivery to improve patient experiences and reduce complications from routine parenteral delivery. Examining excipient strategies and the impact of processing on biologics shows a variety of methods for managing the behaviour of biologic products, and examination of the common device technologies highlights the relative advantages of each type to fit with the physiological and commercial objectives. Examining the analytical techniques and the importance of understanding the activity and purity of the biologic product allows the development of a more suitable suite of tests to demonstrate the product safety and efficacy. Finally, review of real world case studies show how effective formulations can be developed by bringing together techniques for inhaled and biologic product development.

\section{THE APPLICATION OF BIOLOGICAL PRODUCT ANALYTICAL METHODOLOGIES TO SUPPORT THE DEVELOPMENT OF BIOLOGIC DRY POWDER INHALATION PRODUCTS}

Karen Western, Nick Childerhouse, Sandy Munro, and Sara Sefton

Vectura Group plc, Vectura House, Bumpers Way, Chippenham, Wiltshire, SN14 6FH, United Kingdom

The development of a biologic as a dry powder inhaler (DPI) product presents additional challenges to those already typically anticipated for a small molecule DPI (e.g. consistent and stable emitted dose (ED) and fine particle mass). One of the biggest challenges is ensuring that biologic potency and purity is maintained during the various manufacturing processes and afterwards, throughout the product shelf life and during drug delivery through the DPI. The application of a content assay method during development of a spray dried cytokine powder product identified that stabilising excipients were required in the feedstock to protect the cytokine from shear forces during spray drying. Development of a powder matrix for an immunomodulatory peptide required use of a purity (size exclusion chromatography (SEC)) method to detect degradation because a measure of activity alone by enzymelinked immunosorbent assay (ELISA) would not have differentiated between different the stabilising properties of the powder matrices. A further case study for an immunomodulatory protein demonstrated how two different purity methods (SEC and non-reducing sodium dodecyl sulphate-polyacrylamide gel electrophoresis (SDS-PAGE)) may be required to detect aggregation. The final case study demonstrated how, during development pharmaceutics type studies to assess the DPI delivery system, the use of methods to both quantify biologic deposits on the device and assess the stability of these deposits using a biologic product analytical method to determine purity, provided information on formulation and delivery device optimisation. These investigations demonstrate how orthogonal biological product analytical methods have aided formulation/product development, manufacturing feasibility and product stability for potential biologic DPI products.

\section{A NEW DOSING SYSTEM TO FILL DRY POWDER INHALER DISCS}

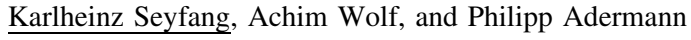

Harro Höfliger Verpackungsmaschinen GmbH, Helmholtzstr. 4, D71573 Allmersbach im Tal, Germany
Several multi-unit dose dry powder inhalers on the market or in development contain injection-molded annular rings, which carry the single powder doses in closed cavities. These pockets can be round or oblong shaped and may be arranged in symmetric or asymmetric circles or even spirals. Current micro dosing systems like dosator, vacuum drum or membrane filler, which are suited to dose powders for inhalation, possess certain limitations when being used to fill inhaler discs, like insufficient robustness, inadequate output or limited flexibility regarding filling degree. Based on the membrane filling technology a new dosing system to fill such discs has been developed. Discs with any number or shape of cavities can be filled in one process cycle, and only two format parts have to be exchanged to adapt the filler to new discs. The system can be integrated into automated machines to fill, assemble and package discbased DPI and it can be up-scaled by simply increasing the number of filling heads.

The dosing system described in this paper has been tested using different lactose monohydrate powder blends with moderate to very poor flow properties, characterized by Carr's indices of $14 \%$ to $42 \%$. The fill weights achieved using $12 \mathrm{~mm}^{3}$ dosing chambers were in the range of $6,3 \mathrm{mg}$ to $9,1 \mathrm{mg}$, the relative standard deviation ranged between $1,7 \%$ and $4,8 \%$. The compaction of the powder during the dosing step was low demonstrating the disc fillers suitability for processing powders for inhalation.

\section{AEROSOLIZATION, DRUG PERMEATION AND CELLULAR INTERACTION OF LEUCINE COATED COMBINATION DPI POWDERS}

Janne Raula $^{1}$, Ville Vartiainen ${ }^{1}$, Esko I. Kauppinen ${ }^{1}$, Luis M. Bimbo ${ }^{2}$, and Jouni Hirvonen ${ }^{2}$

${ }^{1}$ Department of Applied Physics, Aalto University School of Science, Espoo, Finland

${ }^{2}$ Division of Pharmaceutical Chemistry and Technology, Faculty of Pharmacy, University of Helsinki, Finland

\section{Summary}

We investigated the effect of L-leucine coating on particle integrity, aerosolization properties, cellular interaction, viability and drug permeation properties of combination drug powder particles (beclomethasone dipropionate and salbutamol sulphate) for dry powder inhalation. The powder was prepared using an aerosolbased gas-phase method to combine a water-soluble $\beta$-agonist drug, salbutamol sulphate, and corticosteroid, beclomethasone dipropionate, into fine particles which are subsequently encapsulated and coated with L-leucine. Particle morphology studies showed that crystalline leucine coating resulted in intact separated particles. Dissolution profiles showed a faster dissolution of beclomethasone dipropionate, while leaving the salbutamol dissolution unhampered when compared with their physical mixture. Permeation of beclomethasone dipropionate across a differentiated Calu-3 cell monolayer was increased with the L-leucine coating, and time-dependent recrystallization of the drug on top of the Calu3 cell monolayer was also observed. The L-leucine coated particles were further investigated for cytocompatibility in three different pulmonary (Calu-3, A549 and BEAS-2B) and one human macrophage (THP-1) cell lines showing excellent tolerability. The L-leucine coated particles also elicited low reactive oxygen species generation in pulmonary BEAS-2B and macrophage THP-1 cell lines. The inhalable drug powders coated with leucine offer thus an interesting alternative for the delivery of poorly soluble drugs to the lung. 
18. DESIGN-OPTIMIZATION OF DRY POWDER INHALERS: SELECTING AN OBJECTIVE FUNCTION

\author{
Thomas Kopsch $^{1}$, Digby Symons ${ }^{1}$, and Darragh Murnane ${ }^{2}$ \\ ${ }^{1}$ University of Cambridge, Trumpington Street, CB2 1PZ, UK \\ ${ }^{2}$ University of Hertfordshire, College Lane, AL10 9AB, UK
}

Summary

In passive dry powder inhalers (DPIs) drug is often stored in blisters incorporated in the device. The patient inhales through the device, forcing an airstream through the blister. Drug is entrained into the airstream for delivery to the lung. The entrainment and dispersion of drug depend on internal inhaler geometry. Relevant design features include the air path in the device and the blister. Two objectives were considered: A) DPI dose emission that is independent of the inhalation profile, such that the emitted dose reaches similar lung regions when two patients inhale with different inhalation profiles; B) Targeting of the emitted drug to specific pulmonary airways for therapeutic applications. For example, for some medical applications a 'bolus' delivery is desired, i.e. most of the drug should leave at a particular instant. We present an optimisation approach for DPI design for these objectives through simulation of dose emission using computational fluid dynamics (CFD). We demonstrate the approach by computationally optimizing a simple 2D DPI. The geometry considered is a blister where air enters through one hole, entrains drug, and exits through another hole. Three parameters characterised the geometry: the separation of the holes, $s$; the width of the outlet hole, $d_{l}$; and the width of the inlet hole, $d_{2}$. A gradient descent method was used to vary these parameters to optimise the DPI. Through the CFD studies a DPI air path geometry was optimised to achieve an ideal 'early bolus' drug delivery that was independent of the inhalation profile.

\section{A GENERIC QBD METHOD DEVELOPMENT APPROACH FOR A GENERIC PMDI- APPLICATION FOR SIRDUPLA ${ }^{\text {TM }}$ UNIFORMITY OF DELIVERED DOSE METHODOLOGY}

\author{
Andy Cooper \\ 3 M Drug Delivery Systems Ltd, Loughborough, \\ Leicestershire LE11 1EP, UK
}

Analytical method development and validation is performed using a generic Quality by Design (QbD) framework for Life Cycle Management. This is analogous to the framework recommended for product development in ICH guidelines. All methodology has a defined Analytical Target Profile (ATP), a set of predefined objectives that defines performance requirements, and are subject to risk management and continuous improvement processes.

The methodology for uniformity of delivered dose (UoDD), or dose content uniformity (DCU), is a Critical Quality Attribute (CQA) of Orally Inhaled and Nasal Drug Products (OINDP). In line with the QbD paradigm, this critical variable was assessed by designed experiments to understand the performance of the Sirdupla ${ }^{\mathrm{TM}}$ generic product versus the Seretide ${ }^{\circledR}$ Evohaler $^{\circledR}$ innovator product (Salmeterol / Fluticasone Propionate, 120 actuations).

The analytical method requires control of critical shake / fire parameters for the priming, dose collection and waste actuations. In this case the priming and dose collection actuations are performed by an analyst. Automation is used only for the waste actuations performed between the stages of container life where the dose collection actuations are performed. Automation could be extended to the priming/collection actuations, and even the sample recovery process, to exercise greater control.
The optimised methodology demonstrates that the Sirdupla ${ }^{\mathrm{TM}}$ pMDI (pressurised Metered Dose Inhaler) product generates comparable UoDD data to the Seretide ${ }^{\circledR}$ Evohaler ${ }^{\circledR}$ innovator product. Method robustness can therefore be established at an early stage in the method and product development lifecycle, delivering methodology demonstrably appropriate for the product lifespan which is subject to continuous improvement processes in line with the QbD paradigm.

\section{CLINICALLY APPROPRIATE TESTING OF DIFFERENT VALVED HOLDING CHAMBER (VHC)-FACEMASK COMBINATIONS INVESTIGATING DELIVERED MASS TO CARINA FOR A WIDELY PRESCRIBED INHALED CORTICOSTEROID DELIVERED BY PRESSURIZED METERED-DOSE INHALER (PMDI)}

\author{
$\underline{\text { Jason Suggett }}{ }^{1}$, Mark Nagel ${ }^{1}$, Heather Schneider ${ }^{1}$, \\ and Jolyon Mitchell ${ }^{2}$ \\ ${ }^{1}$ Trudell Medical International, 725 Third St., London, \\ N5V 5G4, Canada \\ ${ }^{2}$ Jolyon Mitchell Inhaler Consulting Services Inc., \\ 1154 St. Anthony Rd., London, N6H 2R1, Canada
}

\section{Summary}

Background: Laboratory evaluation of VHC-facemask add-ons is ideally undertaken simulating conditions-of-use. We report a study in which VHCs for small child use ( $n=3$ /group) (AeroChamber Plus ${ }^{\circledR}$ Flow-Vu ${ }^{\circledR}$ anti-static/child mask (aAC + ); PocketChamber ${ }^{\circledR}$ (POC); Vortex $^{\circledR}$ (VOR); SpaceChamber Plus ${ }^{\circledR}$ (SP); A2A Spacer ${ }^{\circledR}$ (A2A); Able Spacer $^{\mathrm{TM}}-2$ (AB2)) were evaluated using an anatomical face/ 4 year child oropharynx model (ADAM-III).

Materials and Methods: Each VHC was evaluated out-of-package (OOP), but the non-anti-static devices were also washed with mild detergent / drip-drying (W). Performance was evaluated by breathing simulator (tidal volume $=155-\mathrm{mL}$, inspiratory: expiratory (I:E) ratio $=1: 2$, rate $/ \mathrm{min}=25$ cycles). The facemask was attached to the model face following a 2-s coordination delay post pMDI actuation. The airway was coupled directly to the breathing simulator via an exit filter capturing fine particles that could theoretically penetrate as far as the carina in a patient $\left(F P_{\text {carina }}\right)$. 5-actuations of fluticasone propionate $(50 \mu \mathrm{g}$ FP/actuation ex actuator) were delivered at 30-s intervals. Recovered FP was assayed by HPLC-UV spectrophotometry.

Results: Values of $F P_{\text {carina }}(\mu \mathrm{g} /$ actuation; mean $\pm \mathrm{SD})$ were $10.5 \pm 1.0(\mathrm{aAC}+/ \mathrm{OOP}) ; 4.0 \pm 1.7(\mathrm{POC} / \mathrm{OOP}) ; 0.7 \pm 1.1(\mathrm{VOR} /$ $\mathrm{OOP}) ; 1.0 \pm 0.3(\mathrm{SP} / \mathrm{OOP}) ; 5.0 \pm 1.4(\mathrm{SP} / \mathrm{W}) ; 0.6 \pm 0.1(\mathrm{~A} 2 \mathrm{~A} / \mathrm{OOP})$; $4.1 \pm 0.9(\mathrm{~A} 2 \mathrm{~A} / \mathrm{W}) ; 0.3 \pm 0.1(\mathrm{AB} 2 / \mathrm{OOP}) ; 4.8 \pm 0.9(\mathrm{AB} 2 / \mathrm{W})$.

Conclusions: Significantly more FP was delivered to the model 'carina' from the aAC + VHC (1-way ANOVA; p < 0.001); associated primarily with decreased VHC retention of medication. Large differences in delivery efficiency may exist when using different VHC-facemask delivery systems. Some of the difference can be eliminated by pre-conditioning non-anti-static devices before first use, however even when this is performed significant differences still exist.

\section{CO-SPRAY-DRIED MANNITOL WITH POLYETHYLENE GLYCOL (PEG) FOR DRY POWDER INHALATION -THE EFFECT OF PEG ON THE AEROSOLIZATION PERFORMANCE AND THE PHYSICAL STABILITY}

Mitsuhide Tanimoto, Atsutoshi Ito, Michiko Kumon, Kazuhiro Inoue, Shuichi Yada, and Hidemi Minami

Formulation Technology Research Laboratories, Daiichi Sankyo Co., Ltd., 1-2-58, Hiromachi, Shinagawa-ku, Tokyo 140-8710, Japan 
Mannitol is one of the excipients widely used in the pharmaceutical industry and approved for pulmonary administration. Recent studies have indicated that polymorphism of carrier particles is one of the possible important factors affecting the performance of DPI formulations. Therefore, for the development of carrier particles for DPI formulation, controlling the polymorph of a carrier particle is considered an important issue. Previously, we developed a manufacturing method for producing a metastable $\alpha$-mannitol by co-spray-drying mannitol aqueous solution combined with polyethylene glycol (PEG). The aim of this study was to evaluate the aerosolization performance and physical stability of co-spray-dried mannitol (co-SDM) with PEG as a carrier particle candidate in a DPI formulation. As results of stability studies of co-SDMs with PEG confirmed, the $\alpha$-mannitol was stabilized during storage when an adequate amount of PEG was added. The molecular weight of PEG little affected the physical stability of mannitol as long as an adequate amount of PEG was added. It was considered to be important to reduce the mobility of mannitol molecules by adding PEG to the co-SDM to stabilize the $\alpha$-mannitol in the co-SDM. As results of in vitro deposition studies further confirmed, as carrier particles, co-SDM particles with 5\% PEG 4000 showed the superior aerosolization performance to that of commercial $\beta$-mannitol. The polymorph of carrier mannitol particles may be shown to be one of the factors that will increase the aerosolization performance. CoSDM particles have shown to be the promising candidate as a carrier particle for DPI formulation.

\section{EVALUATION OF THE AERODYNAMIC PERFORMANCE OF FORMOTEROL DRY POWDER USING DIFFERENT CAPSULES FOR INHALATION: HYPROMELLOSE VERSUS GELATIN FROM QUALICAPS $^{\circledR}$ AND CAPSUGEL $^{\circledR}$}

\author{
Nathalie Wauthoz ${ }^{1}$, Ismaël Hennia ${ }^{1}$, Michel Deleers ${ }^{1}$, \\ Fernando Diez ${ }^{2}$, and Karim Amighi ${ }^{1}$ \\ ${ }^{1}$ Laboratory of Pharmaceutics and Biopharmaceutics, Université \\ libre de Bruxelles (ULB), Boulevard du triomphe CP207, \\ Brussels 1050, Belgium \\ ${ }^{2}$ Qualicaps Europe S.A.U., Avenida Monte Valdelatas 4, \\ Alcobendas 28108, Spain
}

\section{Summary}

Background: The capsule is an important part in the functioning of capsule-based dry powder inhalers (DPIs): it participates in the packaging of the formulation, the aerosolization of the powder and the dispersion of the micronized drug from the carrier after the patient has pierced the capsule and inhaled through the DPI to usually spin it. Moreover, humidity is a parameter that can impact the dry powder and therefore its aerodynamic performance.

Methods: This study evaluated the impact of different kinds of capsule material (gelatin or hypromellose) from different suppliers (Qualicaps ${ }^{\circledR}$ or Capsuge ${ }^{\circledR}$ ) stored in different conditions (usual: $20^{\circ} \mathrm{C}$ $50 \% \mathrm{RH}$; or extreme: $40^{\circ} \mathrm{C} 75 \% \mathrm{RH}$ for $4 \mathrm{~h}$ ) on the fine particle dose (FPD) of a formoterol-based blend from the Axahaler ${ }^{\circledR}$ DPI using the Next Generation Impactor $(100 \mathrm{~L} / \mathrm{min}$ for $2.4 \mathrm{sec}, 10$ capsules/test in triplicate).

Results: The kind of capsule material (gelatin or hypromellose) and the storage conditions $\left(20^{\circ} \mathrm{C} 50 \% \mathrm{RH}\right.$ or $4 \mathrm{~h}$ at $\left.40^{\circ} \mathrm{C} 75 \% \mathrm{RH}\right)$ affected the FPD significantly $(\mathrm{p}<0.001$, three-way ANOVA) whereas the supplier (Qualicaps ${ }^{\circledR}$ or Capsugel ${ }^{\circledR}$ ) did not. The best results were obtained with hypromellose capsules stored at $20^{\circ} \mathrm{C} 50 \%$ RH $\left(3.9 \pm 0.5 \mu \mathrm{g}\right.$ for Qualicaps ${ }^{\circledR}$ and $3.8 \pm 0.4 \mu \mathrm{g}$ for Capsuge $\left.{ }^{\circledR}\right)$. Extreme condition (i.e. $4 \mathrm{~h}$ at $40^{\circ} \mathrm{C} 75 \% \mathrm{RH}$ ) affected the FPD, with a decrease of $\sim 25 \%$, which was not due to the increase in the for- moterol retention in the capsules (maximum 2\%), but was certainly due to the increase in capillary forces between the micronized drug and the lactose carrier.

Conclusion: The choice of the kind of capsule material and the storage conditions are crucial to obtaining optimal aerodynamic performance.

\section{COMPARATIVE IN VITRO PERFORMANCE OF VALVED AND VALVE-LESS, DURABLE AND DISPOSABLE SPACERS}

\author{
Mark Sanders and Ronald Bruin
}

Clement Clarke International Limited, Edinburgh Way, Harlow, Essex, CM20 2TT, United Kingdom

Background: Valved holding chamber (VHC)/spacer devices help mitigate poor pressurised metered dose inhaler (pMDI) technique. We have developed a simple, valve-less, fully-recyclable spacer, comprising a paper body and interchangeable plastic end-fittings (DispozABLE Spacer ${ }^{\mathrm{TM}}$ ), and wished to compare its performance with commercially available spacers.

Methods: This new spacer has been compared with rigid plastic reuseable devices (OptiChamber ${ }^{\circledR}$ Diamond VHC and valve-less Nessi ${ }^{\circledR}$ Spacer), and the collapsible, cardboard disposable VHC, Lite-Aire ${ }^{\circledR}$ using in vitro Andersen Cascade Impactor salbutamol sulphate pMDI (Ventolin ${ }^{\circledR}$ and ProAir ${ }^{\circledR}$ ) aerosol performance data. The research focussed on the $12 \mathrm{~L} / \mathrm{min}$ flow rate, relevant to paediatric use.

Results: Initial experiments at $28 \mathrm{~L} / \mathrm{min}$ established that the DispozABLE Spacer delivered salbutamol reproducibly, was comparable to pMDIs alone, and to the plastic-bodied VHC and valve-less spacer. At $12 \mathrm{~L} / \mathrm{min}$, DispozABLE and Nessi (the valve-less Spacers) were not significantly different. DispozABLE Spacer was superior to both plastic and cardboard VHCs (OptiChamber and Lite-Aire) suggesting that, at paediatric flow rates, valve-less spacers perform better than VHCs, and that body-component material is not a main influence.

Conclusions: The general performance of spacers and VHCs conducted in vitro at conditions that do not reflect the low flow rate of young children or the tidal breathing pattern are not likely to be predictive of clinical efficacy. Young children are likely to use VHCs with a low flow, multiple tidal breathing technique rather than a single inhalation, leading to poor drug delivery, and with poor face-mask seal additionally affecting the inspiratory manoeuvre. The current research, comparing delivery at paediatric-representative flow rates, suggests that valve-less spacers perform better than VHCs.

\section{FORMULATION OF BENZOTHIAZINONES IN BOVINE SERUM ALBUMIN NANOPARTICLES}

\author{
Ayasha Patel $^{1}$, Arcadia Woods ${ }^{1}$, Peter Imming ${ }^{2}$, Adrian Richter ${ }^{2}$, \\ Lea Ann Dailey ${ }^{1}$, and Ben Forbes ${ }^{1}$ \\ ${ }^{1}$ Drug Delivery Research Group, Institute of Pharmaceutical Science, \\ King's College London, 150 Stamford Street, London SE1 9NH, \\ United Kingdom \\ ${ }^{2}$ Martin-Luther-Universität Halle-Wittenberg, Wolfgang-Langenbeck- \\ Str.4, 06120 Halle, Germany
}

The development of albumin-based nanoparticle drug delivery systems is an area that has garnered increasing interest over the years. While an injectable formulation (Abraxane ${ }^{\circledR}$ ) has already achieved success, development in terms of pulmonary drug delivery has been limited due to lack of knowledge about how albumin behaves in the lungs. Previous studies on 
the clearance, biocompatibility and biodistribution of albumin nanoparticles have demonstrated their ability to target drug delivery to the lungs. The next step is to examine drug loading of albumin nanoparticle formulations. This study has investigated the interactions of albumin with two novel anti-tuberculosis compounds, IR 20 and IF 274, from a class of drugs called benzothiazinones. Solubilisation studies with these model hydrophobic compounds in albumin solutions showed that an increase in drug surface area promoted additional solubilisation, e.g. from $\sim 40 \%$ to $\sim 70 \%$ of $1 \mathrm{mg}$ of IR20. Benzothiazinone (BTZ)-loaded albumin nanoparticles were successfully manufactured (size $\sim 100 \mathrm{~nm}$ ) from the solutions, illustrating how compounds that interact with albumin can be incorporated into a nanoparticulate albumin system for inhaled drug delivery.

\section{MANIPULATION OF SPRAY-DRIED COMPOSITE PARTICLES PHYSIOCHEMICAL PROPERTIES AND IN-VITRO PERFORMANCE}

\author{
$\underline{\text { C Moura }}^{1,2}$, S Campos ${ }^{1}, \mathrm{~F} \mathrm{Neves}^{1}$, A Aguiar-Ricardo ${ }^{2}$, and E Costa ${ }^{1}$ \\ ${ }^{1}$ Hovione SA, Loures, 2674-506, Portugal \\ ${ }^{2}$ Faculdade de Ciências e Tecnologia, Universidade \\ Nova de Lisboa, Caparica, 2829-516, Portugal
}

Drugs for dry powder inhaler systems are conventionally formulated using a carrier-based approach. However, drawbacks such as poor drug delivery uniformity and efficiency are frequently observed. To overcome these constrains, new particle engineering technologies that enable the manipulation of formulation/process parameters are being explored, namely, the production of carrier-free composite particles using spray-drying (SD) technology.

The current work addresses the use of SD to produce composite particles of trehalose and L-leucine with improved aerodynamic performance that are suitable for APIs with low and high water solubility, by focusing on fine-tuning the formulation composition and operating conditions. The experiments were conducted based on Quality-by-Design (QbD) principles. All powders were SD according to a Design of Experiments (DoE) in which the formulation: L-Leucine and ethanol percentages, as well as the process outlet drying temperature were manipulated. The impact of the formulation/process variables on the powder properties (particle size, morphology, bulk density, water content) and aerodynamic performance (emitted mass, fine particle fraction (FPF $<4.4 \mu \mathrm{m}$ ) relatively to the capsule fill weight) were evaluated and quantified. A statistical model was implemented to predict the FPF.

Overall, it was demonstrated that the integrated optimization of composition/process conditions can improve the drug delivery efficiency, reaching FPF values of $\sim 70 \%$. It was observed that the ethanol percentage and the outlet temperature were the main contributors for the FPF manipulation, where a direct correlation with the FPF improvement was observed. The leucine percentage had a negligible impact on the powder FPF. The FPF was successfully predicted by a statistical model.

\section{OVERCOMING DIFFERENCES IN PMDI ACTUATOR RESISTANCE TO CREATE A STANDARDISED TRAINING TOOL}

\author{
$\underline{\text { Mark Sanders }}{ }^{1}$, Ronald Bruin ${ }^{1}$, and Cuong $\operatorname{Tran}^{2}$ \\ ${ }^{1}$ Clement Clarke International Limited, Edinburgh Way, Harlow, \\ Essex, CM20 2TT, United Kingdom \\ ${ }^{2}$ i2c Pharmaceutical Services, Cardiff Medicentre, Heath Park, \\ Cardiff, CF14 4UJ, United Kingdom
}

Background: In-Check Flo-Tone ${ }^{\circledR}$ is an approved dual spacer and pressurised metered dose inhaler (pMDI) patient training device that indicates the optimal pMDI actuation point during the inhalation manoeuvre. The device fits many but not all pMDI actuators. A development programme has been undertaken to tailor the existing device to a range of pMDIs that vary in mouthpiece configuration and actuator resistance.

Methods: In this study Flutiform ${ }^{\circledR}$ Landmark $^{\circledR}$ pMDI (FL, $5 \mu \mathrm{g}$ formoterol fumarate $/ 125 \mu \mathrm{g}$ fluticasone propionate, Napp Laboratories) was tested alone or in combination with machined or moulded adaptors paired with either standard Flo-Tone or Flo-Tone development devices: anti-static plastic, a short device, and a short, flared mouthpiece single unit prototype (the Flo-Tone $C R^{\mathrm{TM}}$ ). Aerosol particle size distribution and dose characteristics were derived from Next Generation Impactor experiments conducted at $30 \mathrm{~L} / \mathrm{min}$.

Results: In all assessments, formoterol and fluticasone data trends were the same. Fine particle fraction (FPF) and fine particle dose (FPD) data were comparable between FL alone and the moulded adaptor plus short Flo-Tone device combinations. The formoterol FPF $(\%)$ and FPD $(\mu \mathrm{g})$ values were $50.43 \pm 2.14$ and $2.19 \pm 0.14$ for FL; $50.75 \pm 1.04$ and $2.13 \pm 0.06$ for the short Flo-Tone; and $50.22 \pm 3.10$ and $2.43 \pm 0.03$ for Flo-Tone $C R$. Preliminary data with the Flo-Tone $C R$ to determine reed whistle vibration and detectable whistle flow rates across a range of commonly used pMDIs, suggest that the actuation indicator sounded at $20-25 \mathrm{~L} / \mathrm{min}$.

Conclusions: This development programme has shown that it is possible to tailor an existing audible training aid to a broader range of pMDIs and actuator designs without drug delivery compromise.

\section{A SIMPLE IN-VITRO DEPOSITION SYSTEM TO UNDERSTAND THE ROLE OF INHALED PARTICLE CHARACTERISTICS ON THEIR FATE IN THE LUNG}

\author{
$\underline{\text { Emanuela Cingolani }}{ }^{1}$, Snow Stolnik-Trenkic ${ }^{1}$, Robyn C. Sadler ${ }^{2}$, \\ and Cynthia Bosquillon ${ }^{1}$ \\ ${ }^{1}$ School of Pharmacy, University of Nottingham, Nottingham, \\ NG7 2RD, United Kingdom \\ ${ }^{2}$ GlaxoSmithKline R\&D, Ware, Herts SG12 ODP, United Kingdom
}

A simple exposure chamber to deposit dry powder particles onto multiple cell layers grown at the air-liquid interface on Transwell ${ }^{\circledR}$ inserts was developed and validated as an in vitro tool to study the fate of inhaled particles in the lung. The system consists of a glass desiccator and the PennCentury ${ }^{\mathrm{TM}}$ dry powder insufflator. Salbutamol sulfate (SS) and indomethacin were used as test dry powders. The system delivered between 0.2 and $1.0 \mu \mathrm{g}$ of SS and indomethacin particles (size range $3-5 \mu \mathrm{m}$ ) per Transwell ${ }^{\circledR}$ insert in a controlled and reproducible manner, depending on the position of the inserts in the deposition chamber and the PennCentury ${ }^{\mathrm{TM}}$ drug loading. Transepithelial electrical resistance (TEER) and lucifer yellow flux measurements confirmed that the integrity of Calu-3 cell layers was maintained after exposure to the particle sprays. The transepithelial transport of salbutamol and indomethacin was measured in solution and after particle deposition onto the cell layers. The apical to basolateral $(\mathrm{A} \rightarrow \mathrm{B})$ flux of both drugs delivered to the cell layers as dry powders was significantly higher than when they were applied as solutions. The percentage of drug transported was not dependent on the applied dose in either case but as expected, was greater for the high permeability drug indomethacin. Despite limitations, the deposition system proved helpful in understanding the role of drug particle characteristics on their dissolution/absorption behaviour at the airepithelium interface. 


\section{TRACHEAL DOSE DELIVERY IN A REPRESENTATIVE ORO NASAL AIRWAY MODEL OF A NEWBORN (RONAN) ACROSS THREE NONINVASIVE VENTILATION INTERFACES FOR THE PEDIATRIC POPULATION}

\author{
Andrew O'Sullivan, Louise Sweeney \& Ronan Mac Loughlin
}

Aerogen, IDA Business Park, Dangan, Galway, Ireland

The pediatric population has a preference for noninvasive methods of aerosol drug delivery. Nasal cannula and facemask are two means of noninvasive ventilatory support. This study investigated the effect of these on aerosol delivery to the pediatric patient. The feasibility of aerosol delivery in combination with open and closed facemask options with supplemental oxygen and also along with nasal cannula in a model of a 4 month old was investigated. Tracheal dose at each supplemental oxygen gas flow rate under test (2 and 6 LPM) was recorded in triplicate for each interface type.

Results: The closed face mask facilitated the highest tracheal dose $(19.47 \pm 2.60 \%$ at $2 \mathrm{LPM}) \&(14.40 \pm 0.41 \%$ at $6 \mathrm{LPM})$ compared with both the open face mask $(10.12 \pm 0.86 \%$ at 2 LPM) \& $(6.64 \pm 0.21 \%$ at 6 LPM $)$ and nasal cannula $(6.44 \pm 0.20 \%$ at 2 LPM) \& (1.44 $\pm 0.08 \%$ at 6 LPM). The feasibility of relatively high levels of aerosol drug delivery in a model of a 4 month old paediatric patient was successfully demonstrated. It is evident that closed aerosol delivery systems deliver larger fractions of the aerosol past the trachea than the open systems of open facemask and nasal cannula. It is likely that the closed system facilitates this improved performance on the basis that there is less aerosol lost through leaks as the supplemental oxygen pressurises the system, and during the exhalation phase of the breath.

\section{DIRECT QUANTITATION OF FORMALDEHYDE IN PRESSURISED METERED DOSE INHALER (PMDI) DEVICES}

Mike Ludlow, Kathryn Arthur, Paula Dunster, and Phil Teale

LGC, Newmarket Road, Fordham, Cambridgeshire, CB7 5WW, UK

A direct sampling procedure has been successfully developed for the derivatisation of residual levels of formaldehyde in pressurised metered dose inhaler (pMDI) devices, enabling total quantitation using standard high performance liquid chromatography coupled with ultra-violet detection (HPLC-UV).

The potential presence of formaldehyde, which can be derived from a range of commonly utilised polymeric components, is a particular area of concern for high exposure risk drug delivery systems such as pMDI devices.

Formaldehyde is toxic, allergenic and is a known human carcinogen. The compound is also highly reactive and has the potential to adversely interact with the active pharmaceutical ingredient (API) or other excipient components. This can cause issues in terms of product safety and have a negative impact on the overall efficacy of the drug product.

The short term exposure limit for formaldehyde is under review and is likely to be reduced from the current level of 2 parts per million (ppm). In order to assess the viability of current sampling methodologies a study was performed where placebo pMDI devices were spiked with known amounts of formaldehyde around the level of concern. The results from this study showed that existing procedures would not be suitable.

The developed method has been fully validated in accordance with $\mathrm{ICH}$ guidelines and is capable of sub $\mu \mathrm{g} /$ canister detection levels, in a range of generic drug product formulations, with a limit of detection (LOD) of $0.55 \mu \mathrm{g} /$ canister and reproducibility typically $<2 \%$ RSD.

\section{CARRIER-BASED DRY POWDER INHALATION PART I: IMPACT OF CARRIER MODIFICATION ON CAPSULE FILLING PROCESSABILITY AND IN VITRO AERODYNAMIC PERFORMANCE}

\author{
S Zellnitz ${ }^{1}$, E Faulhammer ${ }^{1}$, V Wahl ${ }^{1}, \mathrm{~J} \mathrm{G} \mathrm{Khinast}^{2}$, and A Paudel ${ }^{1}$ \\ ${ }^{1}$ Research Center Pharmaceutical Engineering GmbH, Inffeldgasse \\ 21a/II, Graz, Austria \\ ${ }^{2}$ Institute for Process and Particle Engineering, Graz University \\ of Technology, Inffeldgasse 21a/II, Graz, Austria
}

This study aims to investigate the effect of carrier characteristics and dosator capsule filling operation on the in vitro deposition of mixtures containing salbutamol sulphate (SS) and lactose and mannitol as model carrier materials. The carrier surfaces of lactose and mannitol were modified via wet decantation. The impact of the decantation process on the properties of carriers was investigated by laser diffraction, density and powder flow measurements, N2 physisorption, small and wide angle X-ray scattering (SWAXS) and scanning electron microscopy (SEM). Differences in carrier type and untreated and decanted materials were identified and the SAXS measurements proved to be a promising technology confirming the successful removal of fines. Adhesive carrier API mixtures with carrier-to-API ratio of 99:1 wt $\%$ were prepared, mixture homogeneity was tested and subsequently the mixtures were filled into capsules at different process settings. Finally, the influence of the decantation process on the in vitro performance of the adhesive mixtures was tested with a next generation impactor. For lactose, the decantation decreased the fine particle fraction (FPF) of SS, whereas the FPF of mannitol as a carrier was only affected by the capsule filling process.

In summary, the DPI formulation based on untreated lactose, especially by capsule filling using a dosing chamber to powder layer (compression) ratio of $1: 2$, proved to be superior in terms of the dosing accuracy (RSD $<0.8 \%$ ) and the in vitro aerodynamic performance (FPF of $12 \%$ ).

\section{CARRIER-BASED DRY POWDER INHALATION PART II: IMPACT OF CARRIER MATERIAL AND API PROCESSING ON THE INTER-PARTICULATE SURFACE INTERACTIONS IN ADHESIVE MIXTURES FOR INHALATION}

\author{
$\underline{\text { S Zellnitz }}{ }^{1}$, E Faulhammer ${ }^{1}$, V Wahl ${ }^{1}$, H Schroettner $^{2}$, \\ $\mathrm{J} \mathrm{G} \mathrm{Khinast}^{3}$, and A Paudel ${ }^{1}$ \\ ${ }^{1}$ Research Center Pharmaceutical Engineering GmbH, \\ Inffeldgasse 21a/II, Graz, Austria \\ ${ }^{2}$ Austrian Centre for Electron Microscopy and Nanoanalysis, \\ TU Graz, Steyrergasse 17/III, Graz, Austria \\ ${ }^{3}$ Institute for Process and Particle Engineering, Graz University \\ of Technology, Inffeldgasse 21a/II, Graz, Austria
}

The aim of this work is to understand the impact of carrier material and API processing on the preparation of adhesive mixtures for inhalation. Additionally, a surface phenomenon, that was observed in a previous work, where spray dried salbutamol sulphate seemed to fuse with the surface of mannitol (Pearlitol 160C), is investigated further. Therefore, mixtures with two different mannitol carriers (Pearlitol 160C, Pearlitol 300D) and spray dried and micronized salbutamol sul- 
phate as model active pharmaceutical ingredient (API) were prepared. Spray dried and micronized API were chosen as these two techniques generate particles with different surface properties. To evaluate the morphology of the mixtures scanning electron microscopy (SEM) images were taken and the air permeability was determined as parameter to detect changed in the powder mixtures resistance to airflow.

Through this study, it was found that the observed surface fusionlike phenomenon is specific for P160C and spray dried (amorphous) salbutamol sulphate. When using micronized API (which is largely crystalline), no fusion of API and carrier surface occurred with both mannitol grades used.

Moreover, this study reveals an exciting observation that selection of suitable mannitol grade enable an efficient surface adherence of spray dried particles of salbutamol sulphate-like API. Our ongoing work is focused on further elucidation of the observed interesting finding on the carrier specific surface interaction behaviour of API.

The next steps will also be the determination of the fine particle fraction (FPF) for the different mixtures, with the special focus on the spray dried API and different mannitol system.

\section{MICRODOSING AND FEEDING OF INHALATION POWDERS VIA A VIBRATORY SIEVE - CHUTE SYSTEM}

\author{
E Faulhammer $^{1}$, M O Besenhard ${ }^{1}$, M Llusa $^{1}$, S Biserni $^{2}$, \\ V Calzolari $^{2}$, A Ferrari ${ }^{2}$, A Paudel ${ }^{1}$, and JG Khinast ${ }^{1}$ \\ ${ }^{1}$ Research Center Pharmaceutical Engineering, Inffeldgasse 13, 8010, \\ Graz, Austria \\ ${ }^{2}$ MG2, Via del Savena, 18. I-40065 Pian di Macina di Pianoro, \\ Bologna, Italy
}

\section{Summary}

This study describes a powder dosing system with a vibratory sieve mounted on a chute that doses particles into a capsule. Vertical vibration occurred within a broad range of frequencies and amplitudes. During dosing events, the fill weight was accurately recorded via a capacitance scale, making it possible to analyze filling characteristics, i.e., the fill rates and their robustness. The results show that fill rate robustness varied distinctly in the operating space. The performed micro dosing studies exhibit that fill rate robustness is of upmost importance to achieve accurate (micro) dosing. Prior to every dosing study, the operating space, i.e., settings that yield a steady flow of powder into the chute, was determined for three inhalation carriers. The performed low-dose studies with the original Microdose system show that capsules can be filled with doses in the range of $2,5 \mathrm{mg}$ and a fill weight variability below $5 \%$ in less than one second. If dosing time is not an issue, the proposed system can dose with almost any accuracy, limited only by the accuracy of the dynamic scale.

It could be demonstrated that such a vibratory sieve chute system is also suitable for continuous (micro-) feeding applications and therefore, MG2's Microdose was rebuild for microfeeding studies. Initial results reveal that robust feeding rates of less than $5 \mathrm{mg} / \mathrm{h}$ are feasible.

\section{PERMEABILITY AS A PERFORMANCE DESCRIPTOR OF DRY POWDER INHALATION CARRIERS: INVESTIGATION OF SEVERAL LACTOSE GRADES}

\author{
Ahmed O. Shalash $^{1}$, Abdulla M. Molokhia ${ }^{1}$, \\ and Mustafa M.A. Elsayed ${ }^{2}$
}

\footnotetext{
${ }^{I}$ European Egyptian Pharmaceutical Industries, Alexandria, Egypt

${ }^{2}$ Department of Pharmaceutics, Faculty of Pharmacy, Alexandria University, Alexandria, Egypt
}

\section{Summary}

Background: Dry powder inhalers (DPIs) have captured the interest of several research groups, for the purpose of solving the performance prediction dilemma. Several studies reported linear inverse relationship between permeability and inhalation performance, while others found no simple relationships. The carrier particle sizes used in these studies were different, that may have resulted in viewing only a part of the whole pattern in each study.

Aim: This study aimed to investigate the relationship between the carrier permeability and the performance of the DPIs and to utilize this relationship in formulation development.

Methods: We prepared inhalation mixtures from six different lactose grades. Carriers and inhalation mixtures were characterized for their particle size distribution using laser diffraction, crystallinity using differential scanning calorimetry, particle shape using image analysis, moisture contents using loss on drying, pore size distribution and permeability using mercury porosimetry. We assessed the in vitro performance of the inhalation mixtures.

Results: The results show that carriers were crystalline with low moisture contents. However, carriers differed in their size distributions, permeabilities and performance $\left(\mathrm{FPF}_{8.06} 5-21 \%\right)$.

Conclusions: Initially at low permeabilities, the increase in performance was associated with increasing carrier permeability, until an optimum performance was reached. At higher permeabilities, a decrease in performance was associated with increasing carrier permeability. The findings explain reported controversies between carrier permeability and performance. Carriers with excessive amount of fines become highly resistant to air flow with lower dispersion/performance. Permeability can account for carrier size distribution, shape and packing in a more performance-relevant manner, it also allows identifying a carrier's optimum fines content to meet device dispersion requirements.

\section{MODULATING TIGHT JUNCTIONS ON AIRWAY EPITHELIAL CELLS TO ENHANCE PARACELLULAR TRANSPORT OF ANTIFIBROTIC DRUGS}

\author{
Maliheh Ghadiri ${ }^{1}$, Paul Young ${ }^{1,2}$, Brian Oliver $^{1}$, \\ Wolfgang Jarolimek ${ }^{3}$, and Daniella Traini ${ }^{1,2}$ \\ ${ }^{1}$ Woolcock Institute of Medical Research, Glebe, \\ NSW 2037, Australia \\ ${ }^{2}$ Discipline of Pharmacology, Sydney Medical School, \\ University of Sydney, Sydney, NSW 2006, Australia \\ ${ }^{3}$ Pharmaxis Ltd, 20 Rodborough Rd, Frenchs Forest, \\ NSW 2086, Australia
}

\section{Summary}

Background: Hydrophilic molecules cannot cross biological membranes; therefore their transepithelial transport could be significantly enhanced if they could access the paracellular pathway. Tight junctions regulate transit through this route and various tight junction modulators have been studied to open the paracellular route in a reversible manner.

Aim: This study aims to enhance the absorption in the lung of a poorly absorbed hydrophilic compound, specifically PXS25, a stable analogue of mannose-6-phosphate with anti-fibrotic properties, via the paracellular route by dilating the tight junctions of airway epithelial cells.

Experimental method: Calu-3 cells were seeded onto cell culture inserts and cultured at the air-liquid interface for 2 weeks to allow for cell differentiation. The cytotoxicity of modulators was measured using MTS assay. Calu-3 cells were treated for $1 \mathrm{hr}$ with 
each modulators (oleic acid $100 \mu \mathrm{M}, \mathrm{Na}$ caprate $1 \mathrm{mM}$ and $\mathrm{NaCl}$ $3 \%$ ) followed by measuring transport of fluorescein sodium (flu$\mathrm{Na}$ ), a hydrophilic marker molecule, via tight junction and transepithelial electrical resistance. In separate experiments the effects of modulators on transport of PXS25 across treated Calu-3 was also evaluated.

Results: Calu- 3 cells before treatment had a transepithelial electrical resistance (TEER) of $749 \pm 78 \Omega \mathrm{cm}^{2}$ after two weeks in culture. After treatment with the modulators, TEER measurements were $102 \pm 10,64 \pm 3$ and $109 \pm 27 \Omega \mathrm{cm}^{2}$ for oleic acid, Na caprate and $\mathrm{NaCl}$, respectively. Flu-Na, and PXS25 were used to assess paracellular transport across the Calu-3 monolayer after treating cells with tight junction modulators. The transport of flu-Na and PXS25 increased significantly after treatment with tight junction modulators, specifically with $\mathrm{NaCl}$.

Conclusion: The transport of PXS25 a hydrophilic antifibrotic molecule increased by modulating the airway epithelia tight junctions.

\section{SATURATED VAPOUR PRESSURE (SVP) MEASUREMENT OF ETHANOL/HFA BINARY MIXTURES}

B Gavtash $^{1}$, B Myatt ${ }^{1}$, H O'Shea ${ }^{2}$, F Mason ${ }^{2}$, D Lewis ${ }^{2}$, T Church ${ }^{2}$, HK Versteeg ${ }^{1}$, G Hargrave ${ }^{1}$, and G Brambilla ${ }^{3}$

${ }^{1}$ Wolfson School of Mechanical and Manufacturing Engineering, Loughborough University, Loughborough, LE11 3TU, United Kingdom

${ }^{2}$ Chiesi Limited, Bath Road Industrial Estate, Chippenham, Wilts, SN14 OAB, United Kingdom

${ }^{3}$ Chiesi Farmaceutici SpA, Via Palermo, 43122 Parma, Italy

\section{Summary}

Addition of ethanol to propellant based formulations of pMDIs results in saturated vapour pressure alteration which is understood to have an impact on the droplet size of the resulting aerosol cloud ${ }^{1,2,3,4}$. Accurate and simple methods of estimating the saturated vapour pressure are important in developing the next generation of pMDI formulations. Here we report the results of a series of vapour pressure measurements of ethanol/HFA134 and ethanol/HFA227 binary mixtures over a wide range of ethanol concentration and temperature. The outcome shows strong departure from Raoult's law. An empirical expression is developed to capture the nonlinear behaviour of vapour pressure in a compact set of equations as follows:

$$
\begin{aligned}
\prod_{v}^{*}\left(x_{e t h}, T\right)= & \frac{P_{m i x}\left(x_{e t h}, T\right)-P_{e t h}(T)}{P_{H F A}(T)-P_{e t h}(T)} \\
= & \left(1-x_{e t h}\right)\left[1+a x_{e t h}+b x_{e t h}^{2}+c x_{e t h} T\right. \\
& \left.+d x_{e t h}^{3}+e x_{e t h}^{2} T+f x_{e t h} T^{2}\right]
\end{aligned}
$$

Where $P_{\text {mix }}\left(x_{\text {eth }}, T\right)$ denotes the saturated vapour pressure of a mixture of HFA and ethanol with mole fraction $x_{\text {eth }}$ at temperature T. $P_{H F A}(T)$ and $P_{e t h},(T)$ are SVP values of pure HFA and ethanol respectively, which are functions of temperature only. Coefficients in $a-f$ in equation 1 are propellant dependent and are tabulated as follows:

\begin{tabular}{lcccccc}
\hline Coefficient & $a$ & $b$ & $c$ & $d$ & $e$ & $f$ \\
\hline HFA134 & -57.73 & 4.325 & 0.4202 & 0.01125 & -0.005239 & -0.000759 \\
HFA227 & 0.613 & -6.784 & 0.171 & -2.666 & 0.03957 & $-7.149 \times 10^{-5}$ \\
\hline
\end{tabular}

\section{CREATING A DESIGN SPACE FOR THE SCALE UP OF A LOW-DOSE DOSATOR CAPSULE FILLING PROCESS FOR INHALATION PRODUCTS}

\author{
E Faulhammer $^{1}$, M Llusa $^{1}, \mathrm{~S}$ Biserni $^{2}, \mathrm{~V}$ Calzolari $^{2}$, \\ S Lawrence ${ }^{3}$, A Paudel ${ }^{1}$, and JG Khinast ${ }^{1}$
}

${ }^{1}$ Research Center Pharmaceutical Engineering, Inffeldgasse 13, 8010, Graz, Austria

${ }^{2}$ MG2, Via del Savena, 18. I-40065 Pian di Macina di Pianoro, Bologna, Italy

${ }^{3}$ GlaxoSmithKline, New Frontiers Science Park, Harlow, Essex CM19 5AW, UK

\section{Summary}

The objectives of this study were to develop a predictive statistical model for low-fill-weight capsule filling of inhalation products with dosator nozzles via the quality by design $(\mathrm{QbD})$ approach and based on that, to study the effect of scaling up a capsule filling operation of capsule fill weight (1- 45mg) and weight variability (RSD) of typical inhalation carriers. Various controllable process parameters and uncontrolled material attributes of 12 powders were initially screened using a linear model with partial least square (PLS) regression to determine their effect on the critical quality attributes (CQA) (fill weight and weight variability). After identifying critical material attributes (CMAs) and critical process parameters (CPPs) that influenced the CQA, model refinement was performed to study if interactions or quadratic terms influence the model. Based on that, we developed a linear predictive model for fill weight and a model that provides a good approximation of the fill weight variability for each powder group. We validated the model, established a design space for the performance of different types of inhalation grade lactose on lowfill weight capsule filling and successfully used the CMAs and CPPs to predict fill weight of powders that were not included in the development set on lab scale. Finally, the scale up performance was tested for a variety of capsule filling speeds, pre-compression ratios and nozzle diameters. The Design Space developed with the Labby (lab-scale machine) can be safely used to set the process parameters to obtain a specific fill weight in the industrial scale machine.

\section{MULTI-METHODOLOGICAL INVESTIGATION ON THE VARIABILITY IN THE MICROSTRUCTURE OF HPMC CAPSULES}

\author{
Eva Faulhammer ${ }^{1}$, V Wahl ${ }^{1}$, D Markl ${ }^{1}$, A Kovalcik $^{2}$, \\ SM Lawrence ${ }^{3}$, JG Khinast ${ }^{1}$, and A Paudel ${ }^{1}$ \\ ${ }^{1}$ Research Center Pharmaceutical Engineering, Inffeldgasse \\ 13, 8010, Graz, Austria \\ ${ }^{2}$ ICTM, Technical University of Graz, Stremeyergasse 9, 8010, \\ Graz, Austria \\ ${ }^{3}$ GlaxoSmithKline, New Frontiers Science Park, Harlow, \\ Essex CM19 5AW, UK
}

\section{Summary}

The objective of this study was to detect differences in the microstructure (thermo-mechanical transitions of capsule shells and moisture-induced alternation on the physico-mechanical behavior) of three different HMPC hard capsule shells from different suppliers using mechanical, spectroscopic and microscopic and tomographic approaches. Dynamic mechanical (thermal) analysis (DMTA), thermogravimetric analysis (TGA), vibrational spectroscopic, X-Ray scattering techniques as well as environmental scanning electron microscopy (ESEM) and optical coherence tomography (OCT) were 
used. Two HPMC capsules manufactured via chemical gelling (using a gelling agent) and one capsule shell manufactured via thermal gelling were included. Characteristic micro-structural alterations regarding (thermo) mechanical and physical properties relevant to capsule performance and processability that are originating from diverse manufacturing routes and suppliers were thoroughly elucidated with the integration of data obtained from multi-methodological investigation. The physicochemical and physico-mechanical data obtained from a gamut of techniques overall implied that thermally gelled HPMC hard capsule shells could offer an advantage in terms of machinability during Dry powder inhaler (DPI) capsule filling owing to their superior micro- and macroscopic as well as specifically the mechanical stability under dry as well as humid conditions. Thermally gelled capsules seemed to be stiffer than chemically gelled counterparts and exhibit higher glass transition ( $\mathrm{Tg}$ ) values, lower moisture content and highest thermo-mechanical stability.

\section{CFD SIMULATION OF PMDI AEROSOLS IN CONFINED GEOMETRY OF USP-IP USING PREDICTIVE SPRAY SOURCE}

\author{
B Gavtash $^{1}$, HK Versteeg ${ }^{1}$, G Hargrave ${ }^{1}$, D Lewis $^{2}$, \\ T Church ${ }^{2}, \mathrm{G} \mathrm{Brambilla}^{3}$, B Myatt ${ }^{1}, \mathrm{H} \mathrm{O}^{\prime}$ Shea $^{2}$, and F Mason ${ }^{2}$ \\ ${ }^{1}$ Wolfson School of Mechanical and Manufacturing Engineering, \\ Loughborough University, Loughborough, LE11 3TU, \\ United Kingdom \\ ${ }^{2}$ Chiesi Limited, Bath Road Industrial Estate, Chippenham, \\ Wilts, SN14 OAB, United Kingdom \\ ${ }^{3}$ Chiesi Farmaceutici SpA, Via Palermo, 43122 Parma, Italy
}

\section{Summary}

Pressurised metered dose inhaler (pMDI) is the most broadly accepted remedy for asthma. Over decades the pharmaceutical community has aimed to enhance system efficiency by paying particular attention to novel formulation development through experimentation. Fundamental understanding of the underlying physical phenomena that governs the aerosol source characteristics is much less advanced. In this work we describe a CFD simulation of the pMDI plume source. The use of a two-phase flow model and a new atomisation model is illustrated for a formulation with 10:90 \% w/w Ethanol-HFA134 in 50 $\mu l$ valve and actuator nozzle with a diameter of $0.3 \mathrm{~mm}$. The model is implemented within a CFD simulation of the resulting aerosol plume in a USP-IP linking the flow conditions inside the actuator to the plume source characteristics. The CFD result shows how plume velocity slows down over a relatively short distance due to high rate of interaction with inhaled air.

\section{THE FORMULATION DEVELOPMENT OF SIRDUPLA $^{\mathrm{TM}}$ PMDI, A GENERIC VERSION OF SERETIDE $^{\circledR}$ EVOHALER $^{\circledR}$}

\author{
Alex Slowey
}

3M Drug Delivery Systems Division, Loughborough, UK

\section{Abstract}

Sirdupla $^{\mathrm{TM} \mathrm{i}}$ pressurised metered dose inhalers (pMDIs) (test products) have been developed to match the Seretide ${ }^{\circledR}$ Evohaler ${ }^{\circledR i i}$ mid and

\footnotetext{
${ }^{\mathrm{i}}$ Sirdupla is a trademark of Mylan Inc.

${ }^{\mathrm{ii}}$ Seretide ${ }^{\circledR}$ and Evohaler $\left.{ }^{(}\right)$are registered trademarks of Glaxo Group Limited.
}

high strength pMDIs (reference products) containing $25 \mathrm{mcg} / \mathrm{actua}-$ tion of Salmeterol (as Salmeterol Xinafoate) and either 125 or 250 $\mathrm{mcg} / \mathrm{actuation}$ of Fluticasone Propionate.

Following the completion of feasibility studies, the aerodynamic particle size distribution (APSD) for Sirdupla ${ }^{\mathrm{TM}}$ were closely matched with the reference products, for both drug substances, with the exception of throat deposition as measured by Andersen Cascade Impactor (ACI).

Optimisation studies were therefore performed to investigate the potential to reduce the throat deposition observed for the test products. Initial optimisation was performed on the mid strength test product using laboratory scale equipment. The APSD data confirmed that a reduction in throat deposition was observed for the optimised mid strength test product. Although a slight shift in the APSD distribution was noted on plates 3 and 5, the APSD match with the reference product was considered suitable for continued product development.

Following these optimisation studies, Sirdupla ${ }^{\mathrm{TM}}$ pMDI was scaled up to development scale equipment for both the mid and high strength test products, prior to the manufacture of the clinical and primary stability batches.

\section{A DESIGN OF EXPERIMENT APPLIED TO PMDI MANUFACTURE IN ORDER TO EXPLORE THE IMPACT OF THE CRITICAL PROCESS PARAMETERS ON PRODUCT PERFORMANCE}

$\underline{\text { Silvia Belotti, Matthew Edwards, Simon Bryan, and Andrew Brown }}{ }^{\mathrm{a}}$

\author{
Pharmaserve NW, 9 Arkwright Rd, Astmoor Industrial Estate, \\ Runcorn, WA7 1NU, United Kingdom \\ ${ }^{a}$ Andrew.Brown@PharmaserveNorthWest.co.uk
}

Salmeterol Xinofoate (SX) is a long-action $\beta_{2}$ adrenoceptor agonist used in asthma and COPD treatment as a bronchodilator. The aim of this work was to evaluate the effect of specific Critical Process Parameters (CPPs) on the aerodynamic performance of an SX pMDI using a Design of Experiment (DoE). The CPPs chosen were the homogenisation time, the mixer speed and the vessel temperature. The Critical Quality Attributes (CQAs) identified as affecting the product performance were the fine particle dose, emitted dose and the total drug content (TDC).

In this study, the formulation used was kept constant at $99.95 \%$ (w/w) HFA134a and $0.05 \%$ (w/w) SX. The mixing time and the recirculation time prior to product filling were also kept constant both at $30 \mathrm{~min}$. A full factorial design was generated using Minitab (Minitab17 Statistical Software, 2010) to evaluate the CPPs effects.

The aerodynamic assessment of the batches was assessed by Andersen Cascade Impactor $(\mathrm{ACI})$ at $28.3 \mathrm{~L} / \mathrm{min}(\mathrm{n}=10$ shots per ACI). Data such as ACI and TDC for each batch were determined using an appropriate HPLC method in triplicate.

In summary, the use of a DoE increased the process and product understanding. For all of the three CQAs, the highest values (TDC higher than $5.8 \mathrm{mg} / \mathrm{can}$, ED higher than $20 \mu \mathrm{g}$, FPD higher than $9.5 \mu \mathrm{g}$ ) are reached when the CPPs have their highest levels (homogenisation time $36 \mathrm{~min}$, mixer speed $200 \mathrm{rpm}$ and vessel temperature $12^{\circ} \mathrm{C}$ ) but this batch has also an high variability, so further investigations are proposed to confirm the relationship.

\section{ASSESSMENT OF PHYSICO-CHEMICAL PROPERTIES OF AMIODARONE HYDROCHLORIDE ON ALVEOLAR MACROPHAGE RESPONSES IN VITRO}

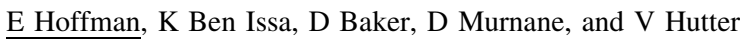

Department of Pharmacy, School of Life and Medical Sciences, University of Hertfordshire, College Lane, Hatfield, AL10 9AB, UK 


\section{Summary}

The development of new inhaled medicines for the treatment of airway disease is being held back by the lack of understanding of how the airways respond to inhaled particulate therapies. A highly vacuolated or 'foamy' alveolar macrophage response is often observed in the lungs of rats when dosed with inhaled particulate candidate drugs which prevents their progression in drug discovery on the grounds of safety, despite not fully understanding if these responses are adverse in humans. It is currently unknown if these alveolar macrophages responses are mediated by the soluble or particulate fraction of these compounds in the lung. The aim of this study was to develop methodology to test if cellular responses to poorly aqueous soluble compounds were caused by the pharmacology of the drug and the soluble fraction entering the cells, or by physicochemical interaction of the insoluble particulate material with the alveolar macrophages. Amiodarone was selected as a model drug as it is a cationic amphiphilic compound established for inducing phospholipidosis in the airways. Concentrations between 1-100 $\mu \mathrm{M}$ amiodarone were prepared and either left unfiltered or filtered through a $0.2 \mu \mathrm{m}$ filter. Solubility studies indicated that $8 \%$ of the $100 \mu \mathrm{M}$ amiodarone preparation was not solubilised, however no significant differences $(p>0.05)$ were observed for viability and phospholipidosis responses in the rat alveolar macrophage cell line, NR8383. Preliminary results indicate that the pharmacological action of amiodarone in its solubilised form primarily mediates alveolar toxicity rather than the interactions of the insoluble particulates with the cells. It is anticipated that similar studies will aid a better understanding of the pathophysiology of airway immune responses to characterise whether the soluble or insoluble fraction of poorly aqueous soluble inhaled drug compounds is responsible for adverse alveolar responses. This will help inform the safety assessment process for new inhaled medicines in drug discovery.

\section{INVESTIGATING THE PERFORMANCE OF INTERACTIVE MIXTURES OF BUDESONIDE AND DIFFERENT MODIFIED GLASS BEADS AS MODEL CARRIERS}

\author{
Niklas Renner ${ }^{1}$, Zinaida Todorova ${ }^{2}$, \\ Regina Scherließ ${ }^{1}$, and Hartwig Steckel ${ }^{3}$ \\ ${ }^{1}$ Department of Pharmaceutics and Biopharmaceutics, \\ Kiel University, Grasweg 9a, 24118 Kiel, Germany \\ ${ }^{2}$ Department of Mechanical Process Engineering, Otto-von-Guericke- \\ University Magdeburg, Universitätsplatz, 2, \\ 39106 Magdeburg, Germany \\ ${ }^{3}$ Deva Holding A.S., Istanbul, Turkey
}

\section{Summary}

In this study glass beads were used as model carriers for a systematic approach to investigate the effect of interparticle interactions on aerosolisation performance, thus deducing their impact on lung deposition. For this glass beads with a median particle size of $500 \mu \mathrm{m}$ were treated with silanes differing in their functional groups to create carrier particles with varying surface hydrophobicity. The degree of hydrophobicity of the resulting product was determined via contact angle measurements. The beads were then blended with spray dried budesonide (BUD) to create interactive mixtures. To obtain different ratios of carrier to active pharmaceutical ingredient (API), blends with different quantities of API were prepared leading to theoretical carrier surface coverages from $25 \%$ to $100 \%$. The aerodynamic performance was investigated using the Next Generation Pharmaceutical Impactor (NGI) and the Cyclohaler ${ }^{\circledR}$ was chosen as a reference dry powder inhaler (DPI) device. Results gained from evaluation of recovered doses showed that a true surface coverage of $100 \%$ could not be reached, regardless of the type of glass beads that were used. This can be attributed to an incomplete interaction between API and carrier leading to loss of API during blending and handling. Nevertheless, an almost linear relationship between true and calculated surface coverage was found. Data obtained from NGI experiments showed a significant increase in fine particle fraction for higher surface coverages, which might be due to the presence of more "active sites" on the carrier surface.

\section{THE DEVELOPMENT OF AN ACTUATOR FOR A NEW GENERIC MDI PRODUCT}

\author{
Stephen Kennedy and Edward Jackson
}

3M DDSD, Morley Street, Loughborough LE11 1EP UK

\section{Summary}

The development of any Metered Dose Inhaler (MDI) presents considerable technical challenges. However, in the development of a generic product, where the emphasis of the project is on matching an existing innovator product, these issues are greatly magnified. An expert knowledge of the influence of each of the many MDI components is critical to achieving a successful project outcome while compressing timelines to be first to reach the market.

This paper discusses the various methods that can be used to assess Innovator actuators such as Computed Tomography and Scanning electron microscopy to enable a matched generic actuator to be produced.

In addition the paper considers key factors for matching pharmaceutical performance such as spray orifice diameter and the ability of a clear component strategy as well as an excellent working relationship with development partners to reducing product development timelines.

The paper concludes that the development of actuators for generic MDIs is a technically challenging process that requires significant expertise. The growing market trend towards lower cost generic products means that companies need experienced partners to develop robust generic products that meet the requirements of the current regulatory landscape. The most qualified partners will be able to demonstrate significant experience of formulating, developing and gaining successful registration of multiple MDI products.

\section{AN OVERVIEW OF THE STATE OF THE ART ON MITIGATING ELECTROSTATIC INTERFERENCE DURING AERODYNAMIC TESTING}

\author{
$\underline{\text { Yang Chen }}^{1}$ and Johan Rene Keegstra ${ }^{2}$
}

on behalf of the Electrostatic Sub-Team of the European

Pharmaceutical Aerosol Group (EPAG)

${ }^{1}$ Respiratory Technology, Woolcock Institute of Medical Research, NSW 2037, Australia

${ }^{2}$ Sandoz International $\mathrm{GmbH}$, Industriestrasse 25, 83607 Holzkirchen, Germany

\section{Summary}

Background: In the pharmaceutical industry, electrostatic phenomena have been a topic of debate and research for many years. It has an impact on formulation performance, especially with powders. In most aerosol formation processes, like emission of droplets from a metered dose inhaler or particles from a dry powder inhaler, electrostatic changing occurs through either contact or friction between different particulates and material surfaces, as well as induction charging. Those electrostatic charges are omnipresent, very difficult to control, almost impossible to eliminate and it can significantly influence particle behavior. 
Materials and Methods: A survey was developed based on the knowledge derived from literature and from expert opinions of the EPAG (electrostatic sub group) members. It consisted of two standardised questionnaires: 1) the effectiveness of different measures and, 2) methods used for minimising electrostatic interference during aerosol testing. A personal email invitation was send to representatives from 7 organisations. The survey asked participants to rank the measures used to reduce electrostatic according to their experience and knowledge, following a score system.

Results: The findings from this preliminary survey showed a lack of information and consensus on how to measure and minimise electrostatics during aerosol analysis, with the majority of the measures currently available not extensively used by pharmaceutical organisations.

Conclusions: How to measure and minimise electrostatics during aerodynamic testing of pharmaceutical is an important issue. Further extensive data will need to be collected to achieve a full overview of this problem and how it should be approached and standardised in the future.

\section{A LEVODOPA WITH L-LEUCINE INHALATION POWDER FOR USE IN PARKINSON'S DISEASE}

\author{
$\underline{\text { M Luinstra }}^{1,2}$, F Grasmeijer $^{2}$, P Hagedoorn ${ }^{2}$, HW Frijlink ${ }^{2}$, \\ and $\mathrm{AH}$ de Boer $^{2}$ \\ ${ }^{1}$ Department of Clinical Pharmacy and Toxicology, Martini Hospital, \\ Groningen, The Netherlands \\ ${ }^{2}$ Department of Pharmaceutical Technology and Biopharmacy, \\ University of Groningen, Groningen, The Netherlands
}

\section{Summary}

Parkinson's disease is a progressive neurodegenerative disorder characterised by degeneration of the dopaminergic neurons in the substantia nigra, causing a lack of dopamine in the striatum. This lack of dopamine causes disruption of motor circuits in the brain resulting in motor function impairments like tremor, rigidity and bradykinesia. Adequate treatment of Parkinson's patients in off periods with orally administered levodopa is thus hindered by a poor bioavailability and a slow onset of effect. Consequently there is a need for a fast and reliable alternative as for instance via pulmonary administration of the drug. We studied the dispersion performance and retention behaviour of various levodopa dry powder formulations in a recently developed high dose inhaler (Cyclops). The in vitro deposition of the most suitable levodopa dry powder formulation was measured at different pressure drops across the inhaler as well as for different dose levels. The objective was to produce a levodopa inhalation powder by means of very simple techniques such as micronisation, either as pure active substance or with the least possible amount of a safe excipient. Levodopa co-micronised with only $2 \% 1$-leucine and dispersed with the Cyclops high dose dry powder inhaler appears to be a promising candidate for the treatment of Parkinson's disease patients in an off period. The combination of this particular formulation and inhaler meets the basic in vitro requirements regarding emission rate, dispersion efficiency and consistency of delivered dose for satisfactory drug delivery to the peripheral airways.

\section{INVESTIGATION OF THE EQUILIBRIUM RELATIVE HUMIDITY OF COMMERCIAL CAPSULE BASED DRY POWDER INHALER PRODUCTS}

\author{
Harsimran Singh Bansal ${ }^{1}$, Gerald Hebbink ${ }^{2}$, \\ Jagdeep Shur $^{1}$, and Robert Price ${ }^{1}$
}

\footnotetext{
${ }^{1}$ Department of Pharmacy and Pharmacology, University of Bath, BA2 7AY, UK

${ }^{2}$ DFE Pharma, Kleverstrasse 187, Goch, Germany
}

\section{Summary}

Hermetically sealing capsule-based dry powder inhaler (DPI) systems at a low relative humidity has numerous benefits such as increased shelf-life, sustained released-dose reproducibility, and a retained expected product performance. Moisture entrapped in hard capsule shells can transfer to the formulation causing an imbalance in the equilibrium conditions achieved under initial packaging giving physicochemical stability issues. If moisture was to egress from the capsule, it would migrate into the powder contained in the capsule and not into the surrounding cavity headspace. Amorphous powders packaged and held under a specific humidity will retain their desired glass transition temperature and hence remain stable over its shelf-life. Equilibration of individual components at a humidity dependant on the characteristics of the powder must occur for a stable marketable product.

Investigations into the headspace humidity of the blistering cavity for several commercial products were carried out to determine packaging, transport and storage requirements for a stable capsule-based DPI. The effect of gelatin capsules on the characteristics of a formulation under different humidities and an elevated temperature of $40^{\circ} \mathrm{C}$ is shown. A formulation named University of Bath (UoB) was prepared and filled into a gelatin capsule. After accelerated stability conditions of $40^{\circ} \mathrm{C} /$ $75 \% \mathrm{RH}$, its initial packaging humidity of $30 \% \mathrm{RH}$ was retained. This suggests that the effect of external environmental conditions are minimised if components are brought together under a common equilibrium state and packaged under these conditions.

\section{EVALUATION OF THE SURFACE FREE ENERGY OF INHALABLE POWDERS}

\author{
J T Pinto $^{1}$, S Zellnitz ${ }^{1}$, M Bresciani $^{1}, \mathrm{~J} \mathrm{G} \mathrm{Khinast}^{1,2}$, E Roblegg $^{1,3}$, \\ and A Paudel ${ }^{1}$ \\ ${ }^{1}$ Research Center Pharmaceutical Engineering GmbH, Inffeldgasse \\ 21a/II, Graz, Austria \\ ${ }^{2}$ Institute for Process and Particle Engineering, Graz University of \\ Technology, Inffeldgasse 13/III, Graz, Austria \\ ${ }^{3}$ Institute of Pharmaceutical Sciences, Department of Pharmaceutical \\ Technology, Karl-Franzens-University of Graz, Universitätsplatz, 4, \\ Graz, Austria
}

A successful dry powder inhalation (DPI) formulation necessitates an in depth understanding of the solid-state, surface and particulate proprieties of powder particles. In particular, surface energy could be critical when developing a successful formulation with the desired inter-particle interaction as these can considerably impact the manufacturing process and pharmaceutical performance.

In the present work, a Wilhelmy plate-based contact angle measurement approach was used in order to assess the nature of the powder surfaces of three model carriers (Inhalac 70, Capsulac 60 and Pearlitol 300 DC) and one model API (salbutamol sulphate). The salbutamol sulphate of inhalable size was produced by jet milling and spray drying and the impact of each technique in particulate energetics was further evaluated. The Good and van Oss equation was used to determine the surface energy and the theoretical work of cohesion of the selected powders and work of adhesion between drug and carrier were calculated according to Young-Dupré equation.

The evaluated carriers showed similar trends for the contact angles measurements between the different used liquids, i.e. the increase in the values of contact angle with the increase in solvent polarity. A marked difference in surface free energy between the milled particles and the spray-dried particles of API was shown. Finally, data on work of adhesion/cohesions suggests that Inhalac 70/spray dried sabutamol sulphate pair may present the most promising outcome regarding aerosolization performance. 


\section{AEROSOL OUTPUT PERFORMANCE OF THE MICRO DEVICE AT HIGH AND LOW TEMPERATURES}

\author{
J Parker, LEA Hardaker, and RHM Hatley
}

Respironics Respiratory Drug Delivery (UK) Ltd, a business of Philips Electronics UK Limited, Chichester, West Sussex, UK

\section{Summary}

The Micro device is being developed for the delivery of aerosolized drugs. We tested 3 Micro devices at $5^{\circ} \mathrm{C}$ and $30^{\circ} \mathrm{C}$ to evaluate the impact of temperature on the performance of the Micro device. The devices were placed in a $5^{\circ} \mathrm{C}$ refrigerator for over 2 hours before being weighed, filled with $300 \mu \mathrm{L}$ of $5 \mathrm{mg} / 2.5 \mathrm{~mL}$ salbutamol sulphate solution equilibrated to $5^{\circ} \mathrm{C}$, and reweighed. The Micro device was connected to a Malvern Spraytec laser diffraction system via a custom connector and was operated until completion of treatment. Aerosolization time was recorded and the device was reweighed before being placed in a $30^{\circ} \mathrm{C}$ incubator for at least 2 hours, and the test was then repeated. This protocol was repeated for each of the 3 devices. The delivered dose and volume median diameter particle size results were consistent across the 3 Micro devices tested and with the 2 different temperatures. Output rate was lower at $5^{\circ} \mathrm{C}$; however, as the delivered dose was unchanged, this only resulted in an increased aerosol delivery time (from 29 seconds at $30^{\circ} \mathrm{C}$, to 39 seconds at $5^{\circ} \mathrm{C}$ ). Overall, the results indicate that the Micro device performance was consistent in the key parameters of aerosol output at temperatures of $5^{\circ} \mathrm{C}$ and $30^{\circ} \mathrm{C}$, which are outside the typical operating range of the Micro device.

\section{INHALABLE GLUCAGON-LIKE PEPTIDE 1 POROUS PARTICLES PREPARED BY SPRAY FREEZE DRYING TECHNIQUE}

$\underline{\text { Sanketkumar Pandya and Amit Misra }}$

CSIR-Central Drug Research Institute, Sector 10, Jankipuram Extension, Lucknow, 226031, India

\section{Summary}

Background: Glucagon-like peptide 1 (GLP-1) is an incretin hormone with multi-faceted actions that find use in the management of type 2 diabetes mellitus. This study focuses on the development and characterization of a GLP-1 loaded dry powder inhalation (DPI) formulation prepared by spray-freeze drying (SFD) technique.

Methods: Inhalable porous GLP-1 particles were prepared by SFD, using a blend of L-leucine and trehalose, and the particles were characterized in terms of particle size distribution and morphology. Carr's index (CI) and angle of repose $(\theta)$, which are measures of powder flow properties, were calculated. In-vitro aerosol performance was assessed in terms of mass median aerodynamic diameter (MMAD), fine particle fraction (FPF) and geometric standard deviation (GSD) using Andersen Cascade Impaction (ACI). The specific surface area $\left(\mathrm{SS}_{\mathrm{BET}}\right)$ of porous particles was determined by nitrogen adsorption-desorption method. The viability of A549 (lung adenocarcinoma) cells after exposure to the DPI was assayed to evaluate the toxic effects of the DPI formulation on alveolar epithelial cells.

Results: Particles prepared in high yield ( $\sim 90 \%)$ by SFD exhibited good dispersibility and aerodynamic performance $(\mathrm{MMAD}=3.7 \pm$ $0.01 \mu \mathrm{m}, \mathrm{FPF}=60.5 \pm 0.5 \%)$. Particles showed good flow properties but poor compressibility. Scanning electron microscopy (SEM) revealed that particles were highly porous structures, and possessed high $\mathrm{SS}_{\mathrm{BET}}\left(5.676 \mathrm{~m}^{2} / \mathrm{g}\right)$ and low density $(0.03 \mathrm{~g} / \mathrm{ml})$. In-vitro cytotoxicity studies on A549 cells indicated that the particles did not exert significant cytotoxicity.
Conclusion: A suitable inhalable formulation of GLP-1 was developed that may be used for the treatment of type 2 diabetes mellitus.

\section{THE DEVELOPMENT OF A SIMPLE AND FAST BIOASSAY FOR POTENCY TESTING OF LPS FOR BRONCHIAL CHALLENGE TESTING}

\author{
$\underline{\text { AJ Lexmond }}^{1}$, I Yilmaz ${ }^{1}$, GW Clarke ${ }^{2}$, CP Page ${ }^{1}$, and BF Forbes ${ }^{1}$ \\ ${ }^{1}$ King's College London, Institute of Pharmaceutical Science, 150 \\ Stamford Street, London, SE1 9NH, UK \\ ${ }^{2}$ Quintiles Drug Research Unit, 6 Newcomen Street, London, \\ SE1 1YR, UK
}

Bronchial provocation with lipopolysaccharide (LPS) has been developed into a clinical challenge model to investigate the effect of novel anti-inflammatory drugs under development for the treatment of diseases such as asthma and COPD that are associated with neutrophil recruitment into the lung. This model has been used for early proof of concept and dose ranging studies in both healthy volunteers and in smoking subjects. However, surprisingly there is no standardised method of LPS delivery to the lungs and several issues have to be addressed before it can be validated as a model to be used more effectively in drug development. These issues mostly relate to a lack of knowledge on the exact dose of LPS that is administered to the lungs in terms of amount and potency (endotoxin strength).

We have investigated whether a bioassay based on the human monocytic cell line (Mono Mac 6) can be developed into a simple and fast tool to determine LPS potency. We investigated various cell densities, LPS concentrations, and incubation times in a 96-well plate format to establish a dose-response relationship between LPS concentration and IL- 6 release from the Mono Mac 6 cells. We found a linear dose-response relationship between $0.05-1 \mathrm{ng} / \mathrm{mL}$ LPS and IL- 6 release at a cell density of $0.5 * 10^{5}$ cells $/ \mathrm{mL}$ and an incubation time of $4 \mathrm{~h}$. The techniques are very simple and the assay can be performed within one day, which with further optimisation may be further shortened to a few hours. In conclusion, we have developed a bioassay that relates Mono Mac 6 IL-6 release to LPS concentration. Ongoing work focuses on reducing the variation between replicate measurements and testing the robustness of the assay using LPS from different strains.

\section{IN VITRO - EX VIVO CORRELATION OF FLUTICASONE PROPIONATE PHARMACOKINETIC PROFILES}

\author{
$\underline{\text { Maria Börjel }}^{1,2}$, Ewa Selg ${ }^{1}$, and Per Gerde ${ }^{1,2}$ \\ ${ }^{1}$ Inhalation Sciences Sweden AB, Kliniskt Forskningscentrum, \\ Hälsovägen 7-9, S-14157 Novum, Sweden \\ ${ }^{2}$ Institute of Environmental Medicine, Karolinska Institutet, \\ Box 210, S-17177 Stockholm, Sweden
}

\section{Summary}

Background: Today there are no available in vitro methods predicting the in vivo pharmacokinetics of inhalation drugs in development. Such methods are needed in both the pharma industry and in academic research. The aim of this work was to compare pharmacokinetic data generated by the promising in vitro dissolution/absorption method DissolvI $t^{\circledR}$ and the ex vivo isolated perfused and ventilated lung of the rat (IPL), which is a well- established experimental model for studying lung pharmacokinetics.

Methods: The PreciseInhale ${ }^{\circledR}$ aerosol system was used to generate and dispense respirable powder aerosols of two formulations containing fluticasone propionate (FP): Flixotide and Flutiform, in pMDI 
canisters. Aerosols from identically actuated inhalers were either deposited on the small circular cover slip glasses used in Dissolvit ${ }^{\circledR}$ or precisely dosed to the IPL perfused in single-pass mode. Analytical quantitation of FP in the samples was performed by LC/MS/MS.

Results: The slight differences in pharmacokinetic profiles of FP in the two pMDI formulations were similarly detected both in the DissolvIt ${ }^{\circledR}$ and IPL systems.

Conclusions: By generating in vivo-like pharmacokinetic profiles with $\mathrm{C}_{\max }$ and $\mathrm{t}_{\max }$, DissolvIt $t^{\mathbb{R}}$ may provide a useful tool for predicting the in vivo pharmacokinetic behavior of different inhalation formulations in development, and be a valuable in vitro dissolution/ absorption method for in vitro - in vivo correlation.

\section{THE EFFECT OF BREATHING PROFILE ON DEPOSITION USING CYSTIC FIBROSIS AND HEALTHY ADULT BREATHING PROFILES DURING NASAL HIGH FLOW OXYGEN THERAPY AT 3 DIFFERENT GAS FLOW RATES}

\author{
Louise Sweeney, Eka Mukeria, Andrew O'Sullivan, and Ronan \\ MacLoughlin
}

Aerogen, IDA Business Park, Dangan, Galway, Ireland

This study investigates the effect of different breathing profiles during Nasal High Flow therapy (NHF). NHF is increasingly used across a variety of patient populations. It has the potential for use for the delivery of bronchodilators and hypertonic saline to the airways. Previously the efficiency of gas flow rates has only been assessed using models of a healthy adult. Here we investigate the effect of gas flow on aerosol delivery efficiency when simulated for both healthy and Cystic Fibrosis (CF) patients during NHF therapy. In this study, we are using Albuterol sulphate as a marker for hypertonic saline solution. Respiratory exacerbations are a persistent recurring effect of the genetic disease, CF. In CF sufferers, the epithelial cells produce a far thicker mucus that clogs passages and cannot be moved easily. This thick mucus gets stuck in the lungs causing breathing difficulties and also leads to infections. Inhalation of hypertonic saline solutions draw water into the mucus, making it easier to clear. We found here, that there is a 9.5 -fold difference in efficiency between the minimum and maximum airflow under investigation for CF sufferers, with the lower airflow being more efficient. This could have substantial implications on patient care, as delivery of hypertonic saline at low gas flow rates could mean a highly efficient yet comfortable means of administration. NHF during $\mathrm{CF}$ treatment at low gas flow rates would mean less irritation, resulting in less coughing and also less interruption to sleeping patterns if delivered continuously overnight.

\section{PERFORMANCE OF THE MICRO DEVICE WITH DIFFERENT INSPIRATORY FLOW RATES}

\author{
J Parker, LEA Hardaker, and RHM Hatley
}

Respironics Respiratory Drug Delivery (UK) Ltd, a business of Philips Electronics UK Limited, Chichester, West Sussex, UK

\section{Summary}

The Micro mesh aerosolization device guides the patient to perform an optimum breathing maneuver through visual signals that inform the patient if their inspiratory flow rate is too fast $(>14 \mathrm{~L} /$ $\min )$ or too slow $(<7 \mathrm{~L} / \mathrm{min})$. We tested the impact of different simulated inspiratory flow rates on the quality of aerosol produced by a Micro device. One Micro device was tested in triplicate with flow rates of 8,10 , or $12 \mathrm{~L} / \mathrm{min}$, when filled with $300 \mu \mathrm{L}$ of $5 \mathrm{mg} /$ $2.5 \mathrm{~mL}$ salbutamol sulphate solution. The inspiratory flow rate was simulated through changes to the extraction flow of a Malvern Spraytec laser diffraction system, and a $9 \mathrm{~L} / \mathrm{min}$ shroud air was set up to prevent recirculation of the aerosol in the Malvern Spraytec. Record capture was started and the Micro device was connected to the Malvern Spraytec via a custom connector. Aerosolization was run into the Malvern Spraytec to completion and the aerosol generation time was recorded. The results showed that there was no change in the mean volume median diameter and very little change in the fine particle fraction ( $\%$ of particles $<5 \mu \mathrm{m}$ ) between the different inspiratory flow rates. In addition, the aerosolization time was equally fast with all 3 inspiratory flow rates, with a mean time of 26 seconds. There was a slight change in the span of the aerosol particle size distribution, which decreased with increasing flow. In conclusion, the particle size of the aerosol from the Micro device was consistent across a range of flow rates representative of the intended normal operating range of the device.

\section{TOWARDS AN IN VITRO BIOASSAY FOR DRUG DELIVERY FROM ORALLY INHALED PRODUCTS}

\author{
Joanna Muddle ${ }^{1}$, Varsha Kanabar ${ }^{1}$, Marc Brown ${ }^{2,3}$, \\ Clive Page ${ }^{1}$, and Ben Forbes ${ }^{1}$ \\ ${ }^{I}$ Institute of Pharmaceutical Science, King's College London, \\ 150 Stamford Street, London, SE19NH, UK \\ ${ }^{2}$ Department of Pharmacy, University of Hertfordshire, Hatfield, \\ $A L 109 A B, U K$ \\ ${ }^{3}$ MedPharm Ltd, R\&D Centre, Units 1 and 3 / Chancellor Court, \\ 50 Occam Road, Surrey Research Park, Guildford, GU2 7AB, UK
}

\section{Summary}

In vivo pharmacodynamic (PD) studies have a number of shortcomings: they are expensive, animal models are not always predictive of effects in humans and clinical studies are not always capable of demonstrating similarities or differences between inhaled products. Therefore, there is a need to develop effective in vitro assays for use as an alternative to in vivo evaluations of the drug action. Using salbutamol sulphate as an archetypal bronchodilator compound, the aim of this study was to develop an in vitro bioassay for drug action following delivery by an orally inhaled product. A cyclic adenosine monophosphate (cAMP) assay was established as a surrogate endpoint for muscle relaxation. When developing the cAMP bioassay, healthy airway smooth muscle (ASM) showed a dose response curve to forskolin, isoprenaline and salbutamol sulphate. The response to salbutamol was most reproducible when drug was incubated with ASM for $15 \mathrm{~min}$. In a co-culture system, salbutamol sulphate was applied to the mucosal surface of an epithelial cell layer (Calu-3 cells) and drug activity was assayed in sub-mucosal ASM after the drug had penetrated the epithelial cell layer. A dose-response was demonstrated in this model which indicated the potential for it to be interfaced with an aerosol deposition technique, e.g. the Twin Stage Impinger (TSI), to evaluate and compare the delivery of $\beta_{2}$ agonists from inhaled products.

\section{INFLUENCE OF CALU-3 PERMEABILITY ON IN SILICO PHARMACOKINETIC PREDICTION OF INHALATION PRODUCTS}

$\underline{\mathrm{S} \mathrm{Wu}}{ }^{1}, \mathrm{~A} \mathrm{Mercuri}^{1}$, S Salar-Behzadi ${ }^{1}, \mathrm{~F} \mathrm{Schiaretti}^{2}, \mathrm{E} \mathrm{Zambelli}^{2}$, G Brogin ${ }^{2}$, M Govoni ${ }^{2}$, C Meindl ${ }^{3}$, S Stranzinger ${ }^{1}, \mathrm{~S} \mathrm{Mohr}^{1}$, N Dzidic ${ }^{1}$, M Bresciani $^{1} \&$ E Fröhlich ${ }^{3}$ 
${ }^{1}$ Research Center Pharmaceutical Engineering GmbH, Inffeldgasse 13, 8010 Graz, Austria

${ }^{2}$ Chiesi Farmaceutici S.p.A., Via Palermo 26/A, 43100 Parma, Italy

${ }^{3}$ Center for Medical Research, Medical University of Graz, Universitätsstraße 27, 8010 Graz, Austria

\section{Summary}

Simulation of pulmonary drug delivery systems is a very complex and challenging process. However, the use of predictive in silico models is always a valuable approach during drug formulation development. The current study was designed to investigate the impact of Calu-3 permeability on the in silico pharmacokinetic (PK) prediction of inhalation products. Formoterol was chosen as model API and its permeability on Calu-3 cells was measured in vitro. An in silico physiologically based pharmacokinetic (PBPK) model of formoterol from an inhaled formulation (Foster ${ }^{\circledR}$ pMDI) was firstly built using Gastroplus ${ }^{\mathrm{TM}}$ (Simulation Plus, Inc.), based on in vitro physiochemical characteristics, physiological input parameters and published in vivo lung deposition data. In order to assess the predictability of the current model without using in vivo lung deposition data, the software calculated lung deposition results based on formulations characteristics were utilized for the PK prediction in the second part of this study. The variation of predicted plasma profiles of marketed inhaled formulations containing formoterol (Foster ${ }^{\circledR}$ pMDI, Symbicort $^{\circledR}$ pMDI and Foradil ${ }^{\circledR}$ DPI) were estimated and compared with in vivo plasma profiles obtained from published clinical data. As shown in this study, using in vitro Calu-3 permeability can help to build and to optimize the PBPK models of inhaled formoterol formulations.

\section{USE OF ADDITIVES IN PARTICLE ENGINEERING OF SPRAY-DRIED NANOSUSPENSIONS}

Katerina Simkova $^{1,2}$, Jasmin Stalder ${ }^{2}$, Felix Stebler ${ }^{1}$, and Berndt Joost ${ }^{1}$

${ }^{1}$ University of Applied Sciences and Arts Northwestern Switzerland, Institute of Pharmaceutical Technology, Gruendenstrasse 40, CH-4132 Muttenz, Switzerland

${ }^{2}$ University of Basel, Department of Pharmaceutical Sciences, Klingelbergstrasse 50, CH-4056 Basel, Switzerland

\section{Summary}

Particle engineering via spray drying is a very useful tool for tuning particles' size, distribution, shape, density, and cohesiveness. Following this concept, dry powders for inhalation comprising large porous particles can be prepared. This is especially useful for pulmonary delivery, where particle size and density of a formulation play major role in delivery efficacy. However, to achieve large geometric size and low density, suitable spray drying additives have to be selected. Spray drying can be advantageously employed to formulate composite nanoparticles-containing microparticles. Embedding nanoparticles into microparticles preserves nanoparticles' benefits, such as improved bioavailability and dose uniformity, and allows their delivery into lungs.

In our work we prepared composite microparticles by spray drying of a drug nanosuspension at two atomising gas settings. Five additives (ammonium carbonate, albumin, glycine, leucine, or trileucine) were used during the spray drying in order to form porous/hollow particles. The effect of these additives on particle size and morphology, surface area, and aerodynamic behaviour was studied. At lower atomising gas setting, the fine particle fraction (FPF) for all excipients except trileucine ranged between 18.9 and $28.5 \%$. Trileucine composite particles reached FPF of $53.0 \pm 2.7 \%$; the mass median aerodynamic diameter (MMAD) was $2.0 \pm 0.2 \mu \mathrm{m}$. Even better results were reached at higher atomising gas setting: all powders except for am- monium carbonate had FPF above 55\%, trileucine having the highest FPF of $68.7 \pm 2.0 \%$.

\section{IN VITRO ASSESSMENT OF ALVEOLAR MACROPHAGE CHARACTERISTICS FOR THE APPLICATION OF INHALED PHARMACEUTICALS}

\author{
A Martin, D Murnane, D Chau, M Brown, and V Hutter
}

Department of Pharmacy, School of Life and Medical Sciences, University of Hertfordshire, College Lane, Hatfield, AL10 9AB.

\section{Summary}

Lung disease such as asthma and chronic obstructive pulmonary disease (COPD) are increasing the global heath burden affecting hundreds of millions of people worldwide. The development of novel inhaled therapies is being hindered by the lack of understanding of alveolar macrophages to inhaled particulate medicines. In animal models, there is increasing evidence that inhaled medicines are taken up by alveolar macrophages resident within the lung resulting in poorly understood perturbations. The aim of this work is to identify an appropriate in vitro alveolar macrophage culture model for the generation of a human co-culture model. This model will allow us to better understand alveolar physiology at a cellular level and predict in vivo responses to pharmaceutical particulates. Human lung, U937 monocyte cell line was differentiated to alveolar macrophages in the presence of phorbal myristate acetate (PMA) and differential characteristics specific to PMA naïve and PMA treated cells were identified. These characteristics include cell viability, immunological changes in surface markers and phagocytic activity. Human U937 monocyte cell line was differentiated to the macrophage phenotype using $(5-500 \mathrm{nM})$, incubation and resting times $(0-72 \mathrm{~h})$. Cells were harvested and differentiation was validated for surface marker expressions by microarray technology, flow cytometry and confocal microscopy. In general, cellular perturbations were identified in alveolar macrophages compared to their phenotype. The U937 cell line has the potential to provide a platform appropriate to explore macrophage responses. Characterisation of a cell line representative of alveolar macrophages will allow for a concrete basis to be established for which a co-culture model can be developed in order to understand alveolar macrophages in response to inhaled pharmaceuticals. Work is ongoing to standardise a protocol for generating alveolar macrophage cells from monocytes and to validate the U937 cell line as a suitable inhaled response model.

\section{PREPARATION OF THEOPHYLLINE INHALABLE PARTICLES BY WET MILLING AND SPRAY DRYING; THE INFLUENCE OF MANNITOL AS A CO-MILLING AGENT}

\author{
M Malamatari $^{1}$, S Somavarapu ${ }^{1}$, M Bloxham $^{2}$ \& G Buckton ${ }^{1}$ \\ ${ }^{1}$ UCL School of Pharmacy, 29-39 Brunswick Square, London, \\ WC1N 1AX, UK \\ ${ }^{2}$ GSK Medicines Research Centre, Gunnels Wood Road, Stevenage, \\ Hertfordshire, SG1 2NY, UK
}

\section{Summary}

This study aims to prepare inhalable theophylline particles by formulating the drug with mannitol. The effect of mannitol as a comilling agent on the micromeritic, solid state properties and aerosolisation performance of the engineered particles was investigated. Theophylline suspensions were produced by wet bead milling in 
isopropanol alone and in the presence of mannitol and were further solidified by spray drying. The spray dried suspension of theophylline consisted of elongated particles of a mean particle size of $4.64 \pm 0.87 \mu \mathrm{m}$. The spray dried suspension of theophylline and mannitol consisted of submicron theophylline particles embedded in a porous mannitol framework, with a mean particle size of $2.18 \pm 0.30 \mu \mathrm{m}$. In both formulations, the drug retained the crystalline state of the raw drug (theophylline anhydrous form I). Inclusion of mannitol during the wet bead milling step of theophylline resulted in formulations which after spray drying exhibited a two-fold increase in the fine particle fraction compared to the spray dried theophylline suspensions without mannitol. The enhanced aerosolisation performance of the spray dried suspensions of theophylline containing mannitol can be attributed to their smaller size, spherical morphology and increased specific surface area that can be used as an indicator of their porosity. Overall, simultaneous wet milling of a drug in the presence of mannitol in an organic solvent followed by spray drying may be an applicable formulation approach for enhancing the aerosolisation performance of (moderately) water-soluble drugs.

\section{THE DEVELOPMENT OF A NOVEL METERED DOSE INHALER CYCLIC OLEFIN COPOLYMER ELASTOMER SEALING SYSTEM}

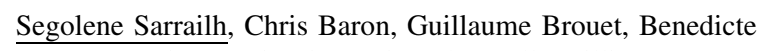
Grosjean, Eric Piazzoni, and Gerallt Williams

Aptar Pharma, Le Vaudreuil, 27100, France

\section{Summary}

The objective of this work was to develop a new and improved sealing system for MDI (metered dose inhaler) products. The sealing of a metered dose valve to a canister in an MDI is a key part of the overall integrity of the MDI system and has historically been most frequently done using nitrile (black or white) or EPDM (ethylene propylene diene monomer) seals. The main MDI sealing system ensuring the container closure between the metered dose valve and the canister could influence many attributes of the resulting MDI including moisture content, leak rate, impurities and degradation products, dose content uniformity, particle size distribution (or fine particle mass) and extractable \& leachables.

The potential for moisture to contribute to instability and particle aggregation in non-aqueous pharmaceutical aerosols formulations is well known ${ }^{[1]}$, and minimizing moisture ingress and content are of interest in order to develop and manufacture stable aerosol formulations. The cleanliness of the materials used in the MDI container closure system, and minimizing extractables and leachables are also seen by regulatory authorities as key elements with regard to both safety ${ }^{[2]}$ and eventual stability of the MDI products ${ }^{[3]}$. In addition any sealing system for MDIs has to have low long term leak rates over a wide range of environmental conditions.

This work describes the development of an innovative sealing material, cyclic olefin copolymer elastomer (COCe), which can offer several advantages in terms of the overall performance of the MDI system, specifically with regard to moisture resistance and cleanliness and thus eventually to the overall stability and performance of such MDI products incorporating this kind of valve to canister sealing technology. Key performance characteristics were studied such as leak rates, moisture ingress, extractables and compatibility with different HFA (hydrofluoroalkane)/ethanol ratios as well as other manufacturing related parameters such as crimping and recycling have also been investigated.

Cyclic olefin copolymers ${ }^{[4]}$ are a new class of polymeric materials with property profiles which can be varied over a wide range during polymerization. These new materials exhibit a unique combination of properties which can be customized by varying the chemical structure of the copolymer. Interesting performance benefits for MDI applications could be considered as - low water absorption, excellent water vapour barrier properties, rigidity, strength and compression set, excellent biocompatibility, good resistance to acids and alkalis and their ability to be moulded into specific component parts. COCs have already found successful industrial applications in the parenteral drug arena and their unique combination of properties, detailed above, make them ideal solutions for applications such as prefilled syringes ${ }^{[5]}$.

\section{ADVANCED IN SILICO MODELING OF THE PERFORMANCE OF INHALATION PRODUCTS}

\author{
Sharareh Salar-Behzadi ${ }^{1}$, Shengqian $\mathrm{Wu}^{1}$, Claudia Meindl ${ }^{2}$, \\ Sandra Stranzinger ${ }^{1}$, Annalisa Mercuri ${ }^{1}$, Stephan Mohr ${ }^{1}$, \\ Massimo Bresciani ${ }^{1}$, Johannes Khinast ${ }^{1}$, and Eleonore Fröhlich ${ }^{1,2}$ \\ ${ }^{1}$ Research Center Pharmaceutical Engineering GmbH, Inffeldgasse \\ 13, Graz, 8010, Austria \\ ${ }^{2}$ Center for Medical Research, Medical University of Graz, \\ Stiftingtalstrasse 24, Graz, 8010, Austria
}

\section{Summary}

In vivo Cmax values and pulmonary absorption of budesonide applied by Turbuhaler ${ }^{\circledR}$ were predicted with GastroPlus ${ }^{\mathrm{TM}}$ software, using either default pulmonary permeability data or in vitro experimental one. The experimental permeability data were gained using Calu-3 cells cultured on polycarbonate membranes (transwells) in submersion (SC) or under air-liquid interface (AIC) conditions to produce mucin. Calu-3 cells in AIC showed lower transepithelial electrical resistance (TEER) and higher permeability to budesonide than cells in SC. The produced mucin in AIC cell cultures did not reduce the transport rate of budesonide.

The experimental permeability data were lower than the calculated pulmonary permeability values by software. The software was able to predict in vivo performance reasonably well and the use of experimental permeability data further improved this prediction.

This work shows the supportive impact of combining advanced in vitro and in silico methods on the efficient modeling of pharmaceutical products in vivo performance. Development of such strong tools is the requirement for promoting safe and fast drug development.

\section{CO-SPRAY DRIED RESVERATROL AND BUDESONIDE MICROPARTICLES: PREPARATION, CHARACTERIZATION AND ANTI-INFLAMMATORY ACTIVITY ON ALVEOLAR MACROPHAGES}

$\underline{\text { Valentina Trotta }}^{1,2}$, Wing-Hin Lee ${ }^{1}$, Ching-Yee Loo ${ }^{1}$, Santo Scalia ${ }^{2}$, Paul M. Young ${ }^{1}$, and Daniela Traini ${ }^{1}$

\author{
${ }^{1}$ Respiratory Technology, The Woolcock Institute of Medical \\ Research, and Discipline of Pharmacology, Sydney Medical School, \\ The University of Sydney, NSW 2006, Australia \\ ${ }^{2}$ Department of Chemical and Pharmaceutical Sciences, University of \\ Ferrara, 44121 Ferrara, Italy
}

Background: Oxidative stress is instrumental in the pathogenesis and progression of chronic obstructive pulmonary disease (COPD). Patients with severe COPD are sometime afflicted by corticosteroid' unresponsiveness due to inhibited histone deacetylase (HADC2) activity in alveolar macrophages. Therefore, novel therapeutic strategies that target macrophages, based on the use of 
antioxidant compounds, could be explored to improve corticosteroids responses in COPD patients. In this study, an inhalable microparticle formulation containing resveratrol (RES) and budesonide (BD) was developed.

Methods: The co-spray dried (co-SD) RES and BD microparticles were produced using a Buchi B-290 Spray dryer and their morphologies and aerosol performances characterized using scanning electron microscopy (SEM) and multi stage liquid impinger (MSLI), respectively. The effect of spray-dried RES and BD, alone and in combination, on cell viability were investigated against a NR8383 alveolar macrophages cell line. The extent of anti-inflammatory activity of RES and BD, alone and in combination was also studied on lipopolysaccharides (LPS) induced NR8383 cells.

Results: The co-SD microparticles of all formulations exhibited morphologies appropriate for inhalation administration, as observed by SEM. Analysis of the deposition profiles showed an increase in aerosol performance proportional to BD concentration. Cell viability assay demonstrated that alveolar macrophages could tolerate a wide range of RES and BD concentrations. In addition, RES and BD were able to decrease the levels of tumor necrosis factor alpha (TNF- $\alpha$ ) and interleukin-6 (IL-6) in LPS induced alveolar macrophages.

Conclusions: Single, co-SD RES and BD microparticles exhibited morphology and aerosol properties suitable for inhalation drug delivery. In vitro studies showed that alveolar macrophages could tolerate concentrations of RES and BD from 1.25 to $80 \mu \mathrm{M}$ and both were able to reduce the levels of TNF- $\alpha$ and IL- 6 in a time dependent manner.

\section{THE INFLUENCE OF INTRINSIC AND EXTRINSIC LACTOSE FINES ON THE PERFORMANCE OF DRY POWDER INHALER FORMULATIONS}

\author{
Ronja Wittmann ${ }^{1}$, Eva Maria Littringer ${ }^{2}$, \\ Eugen Schwarz ${ }^{2}$, and Hartwig Steckel ${ }^{3}$ \\ ${ }^{1}$ Department of Pharmaceutics and Biopharmaceutics, Kiel \\ University, Grasweg 9a, Kiel, 24118, Germany \\ ${ }^{2}$ Meggle Excipients \& Technology, Megglestraße 6-12, Wasserburg, \\ 83512, Germany \\ ${ }^{3}$ Deva Holding AS, Istanbul, Turkey
}

It is a well-known formulation concept to increase the fine particle fraction (FPF) of dry powder inhalers (DPIs) by the addition of lactose fines. Usually during a first mixing step lactose carrier particles and fines are blended. In a second mixing step the API is added and ternary mixtures between the lactose carrier, the lactose fines and the API form. As all commercially available lactose fines are produced by milling little is known about the influence of the origin of the fines and hence the physico-chemical properties of the fines on the DPI performance. For this reason the aim of this work was to study the influence of intrinsic fines, which were prepared during the crystallization of the carrier material, and extrinsic milled fines, which were added to a carrier with a reduced fines content in a separate mixing step, on the performance of a DPI formulation. Adhesive mixtures with a hydrophilic (salbutamol sulfate) and a lipophilic (budesonide) API and three different carrier combinations (lactose RF "reduced fines", lactose IF "intrinsic fines" and lactose EF "extrinsic fines") were prepared and further analyzed.

Only the presence of externally added milled fines increased the FPF. The presence of intrinisic fines did not improve the FPF. The physico-chemical properties of the carrier combinations assessed in this study such as amorphous content and surface energy couldn't be used to predict the performance of the formulation.

\section{UNDERSTANDING THE IMPORTANCE AND EFFECTS OF SHAKING ON PMDI PERFORMANCE}

\author{
Alyssa Newcomb and Dino J. Farina
}

Proveris Scientific Corporation, 290 Donald Lynch Boulevard, Suite 100, Marlborough, MA USA

\section{Summary}

This paper explores how a design of experiments ("DoE") approach was employed to provide a foundational understanding of the effects of shaking on dose content uniformity ("DCU") and spray pattern performance of commercially available albuterol pressurized metered dose inhaler ("pMDI") products. The pMDIs tested were suspension formulations with different excipients that are all known to be sensitive to shaking based on their respective usage instructions for patients (i.e. the instructions only include language such as " ...shake well before each use..."). The DoE focused on controlling and systematically varying the duration, angle, and frequency of shaking immediately prior to automated actuation and measuring the resultant DCU and spray pattern performance of the emitted aerosols. DCU was selected as an obvious output for in vitro performance based on accepted regulatory guidance documents. Specific optical spray pattern measurements were included in the DoE to see if such measurements could be correlated to the shaking conditions, and if so, how these measurements could be used to build an alternative model for efficient, high resolution, in vitro performance prediction for through life testing of pMDIs. The results indicate that the pMDIs tested have statistically significant differences in their performance sensitivities to shaking and that these differences should be explained to patients for optimal benefit.

\section{INVESTIGATING THE EFFECT OF MODIFIED BREATH ACTUATED MECHANISMS ON THE DISPERSION PERFORMANCE OF THE NEXTHALER ${ }^{\circledR}$}

\author{
Alan Tweedie, Francesca Mason, and Dave Lewis
}

Chiesi Limited, Chippenham Research Centre, Chippenham, Wiltshire, UK, SN140AB

Background: Chiesi's NEXThaler ${ }^{\circledR}$ is a dry powder inhaler equipped with a breath actuated mechanism (BAM) which controls dose release in response to pressure drop. In this investigation the BAM was modified to vary the release of formulation and subsequently the airflow velocity into which the dose was dispersed.

Methods: A $4.7 \%$ w/w API:lactose carrier formulation was delivered from four experimentally modified NEXThaler devices, in vitro dispersion performance was assessed using a fast screening impactor (FSI), asthmatic profiles were generated using a breath simulator; a bespoke LiveShot rig was used to understand evacuation kinetics.

Results: The control NEXThaler device required $0.223 \mathrm{~s}$ to reach peak powder discharge, evacuating into a flow rate of $46.3 \mathrm{~L} \mathrm{~min}^{-1}$. With the BAM removed, or the trigger pressure altered the time taken to reach peak evacuation varies, ranging from $0.068 \mathrm{~s}$ to $0.196 \mathrm{~s}$, in turn this influences the powder entrainment flow rate which ranged between $22.0 \mathrm{~L} \mathrm{~min}^{-1}$ and $75.0 \mathrm{~L} \mathrm{~min}^{-1}$. Improvements in dispersion performance (FPD $<5 \mu \mathrm{m}$ ) were observed as the BAM release pressure was increased, the No BAM variant produced the lowest FPD $(129 \mu \mathrm{g})$, whereas the high BAM variant had the highest FPD $(222 \mu \mathrm{g})$.

Conclusion: The velocity of the air has an effect on entrainment and API dispersion performance; this can be controlled using a BAM system, such as that exemplified in the NEXThaler device. 


\section{A FRESH PERSPECTIVE ON HOW PARTICLE SIZE CHANGES LUNG DEPOSITION}

\author{
$\underline{\text { N Stevens }}$ and D Prime \\ GSK, Park Road, Ware, Herts, SG12 ODP, UK
}

The aerodynamic particle size of an inhaled medicine plays a very important role in determining the drug dose delivered to the lung and also how that dose is partitioned between the different types of lung airways. Dose delivered to Bronchi, Bronchioles and Alveolated airways may be expected to result in different pharmacological and clinical outcomes as a result of the differing physiologies of these airway regions. Unfortunately it is not yet possible to directly measure the exact drug dose delivered to each region so inferences are made by comparing pharmacokinetic and pharmacodynamic outcomes with other surrogate measurements of the regional lung dose.

Here it is demonstrated using illustrative examples from the literature how use of a simple mathematical model can help ensure that interpretations of experimental data are consistent with lung deposition physics. These include two validation datasets and one example of a combination Dry Powder Inhaler (DPI) product. Both the math model and the reference experimental literature indicate that particles of all sizes in the range 6$0.5 \mu \mathrm{m}$ deposit in all airway regions to some degree. Changes in particle size within this range cause a quantitative shift in the dose delivered to the larger and smaller airways but there is no qualitative change in the location of deposition. This is at variance with some interpretations of experimental data in the literature, thus the value of referring to a simple math model to obtain a physics-driven analysis is demonstrated.

\section{ION-PAIRS - A NOVEL FORMULATION STRATEGY TO ALTER DRUG DISPOSITION IN THE LUNGS}

\author{
$\underline{\text { Bridie Dutton }}{ }^{1}$, Robyn Sadler ${ }^{2}$, Ben Forbes ${ }^{1}$, and Stuart Jones ${ }^{1}$ \\ ${ }^{1}$ Institute of Pharmaceutical Science, King's College London, 150 \\ Stamford Street, London, SE1 9NH, UK \\ ${ }^{2}$ GlaxoSmithKline, Park Road, Ware, Hertfordshire, \\ SG12 ODP, UK
}

\section{Summary}

The lungs are an attractive route for the administration of both systemically and locally acting medicines for numerous reasons, including their large surface area and relative lack of metabolic activity for many drug molecules. This means of medicine administration does however also have its challenges. The main issue seen with pulmonary drug delivery is the rapid elimination of the active compound, meaning that many medicines must be administered multiple times a day, leading to poor patient compliance. An interesting strategy for altering the post-deposition fate of drug delivered to the lungs by inhalation is the use of ion-pairs. The formation of drug ion-pairs offers an opportunity to alter the biopharmaceutical properties of inhaled compounds without altering their structure or pharmacological activity. In order for a drug to form an ion-pair an excess of counter ion must be used. Thus, existing medicines that contain counter ions, such as salbutamol sulfate, do not take advantage of their ion-pairing potential. In this study the ion-pairing between salbutamol and 3 counter ions (sodium sulfate, sodium gluconate and sodium octanoate) was investigated using Fourier Transformed Infra-red spectroscopy, and it was found that the sodium gluconate complex is stronger than that of sodium sulfate with binding constants of 2.270 and 1.569, respectively. Salbutamol octanoate binding could not be quantified due to the complex nature of the ion-pair formed. The results of this study illustrate how ion-pairs can be formed and characterised in preparation for future pharmacokinetic assessment.

\section{EFFECT OF INHALATION PROFILE AND REALISTIC THROAT GEOMETRY ON AERODYNAMIC PERFORMANCE CHARACTERISTICS OF A DEVELOPMENTAL INHALATION PRODUCT DELIVERED WITH THE GENUAIR ${ }^{\circledR}$ INHALER}

\author{
Georg Wolf and Phillip Krüger
}

Sofotec GmbH, Benzstraße 1-3, 61352 Bad Homburg, Germany

\section{Summary}

A method using realistic inhalation profiles and realistic throat models was developed, verified and applied to more accurately characterise a developmental inhalation product delivered with the Genuair $^{\circledR}$ inhaler. 41 inhalation profiles were recorded and the $10^{\text {th }}$, $50^{\text {th }}$, and $90^{\text {th }}$ percentiles were calculated for peak inspiratory flow rate (PIF; L/min), flow increase rate (FIR; $\mathrm{L} / \mathrm{s}^{2}$ ) and inhalation volume $(\mathrm{V} ; \mathrm{L})$. The three inhalation profiles closest to each of the percentiles for all three parameters were selected (weak, medium, strong). The Universal Induction Port ${ }^{[1]}$ (UIP), the Alberta Idealised Throat ${ }^{[2]}$ (AIT) as well as three anatomically accurate throats ${ }^{[3]}$ were used as throat models. The test setup included an inhaler in an air tight chamber connected to the respective throat and a mixing inlet, fixed between throat and pre-separator of a next generation impactor (NGI). A lung simulator was connected to the airflow between supplementary air and mixing inlet. This lung simulator was used to replay the inhalation profiles to reproduce them at the inhaler. During the study every throat was tested with each of the three profiles in a cross-over design in three replicates. Samples were assayed with a validated HPLC method. The results show that throat models and to a lesser extend the inhalation profiles have an impact on the fine particle dose (FPD), mass median aerodynamic diameter (MMAD), coarse fraction (CF) and throat deposition of the Genuair ${ }^{\circledR}$ inhalation product. Aerodynamic particle size distribution is influenced by different throat geometries rather than inhalation profiles. This test method can provide valuable information about inhalation products during development.

\section{A COMPUTATIONAL FLUID DYNAMICS (CFD) MODEL OF THE START-UP KINETICS OF THE ANDERSEN CASCADE IMPACTOR (ACI)}

\author{
HK Versteeg $^{1}$, P Zhao ${ }^{1}$, C Blatchford ${ }^{2}$, M Copley $^{3}$, \\ D L Roberts ${ }^{4}$, and JP Mitchell ${ }^{5}$ \\ ${ }^{1}$ Wolfson School of Mechanical \& Manufacturing Eng., \\ Loughborough University, Loughborough, LE11 3TU, UK \\ ${ }^{2} 3 \mathrm{M}$ Health Care Limited, 1 Morley Street, Loughborough, \\ LE11 1EP, UK \\ ${ }^{3}$ Copley Scientific Limited, Colwick Quays Business Park, \\ Nottingham, NG4 2JY, UK \\ ${ }^{4}$ MSP Corporation, 5910 Rice Creek Parkway, Shoreview, \\ Minnesota, 55126, USA \\ ${ }^{5}$ Jolyon Mitchell Inhaler Consulting Services, London, \\ Ontario, N6H 2R1, Canada
}

\section{Summary}

Background: The dynamics of flow through the Andersen Cascade Impactor (ACI) at start-up for the performance evaluation of dry powder inhalers (DPIs) are poorly understood. Recent studies by the European Pharmaceutical Aerosols Group (EPAG) investigated the effects of reducing the sample volume on the aerodynamic particle size distributions (APSD) of aerosols generated by DPIs. When the inhaled air volume was significantly reduced below the internal vol- 
ume of a Next Generation Impactor (NGI), size fractionation was incomplete, as expected, and the instrument under-reported the fine particle dose. Surprisingly, the same effect was much less severe with the ACI. The present study investigated, from a theoretical perspective, the likelihood that non-uniform particle transport is taking place in this impactor.

Materials \& Methods: We report the results of a computational fluid dynamic (CFD) investigation of the transient flow through a model of the ACI to confirm the existence of flow path channelling that could explain the mass-per-stage data observed previously.

Results: The CFD results confirmed that a negative pressure wave propagates back from the vacuum source at the ACI exit through the CI. Air flow from the inhaler into the ACI is initiated when the suction wave reaches the induction port.

Conclusions: The predicted start-up kinetics compare favourably with measurements of the air flow as a function of time. Complex regimes exist in the upper stages of this CI, involving jets that provide short-circuits, and which are therefore believed to be responsible for the experimentally observed more rapid particle transport through the ACI.

\section{ACOUSTIC EMISSION CHARACTERIZATION OF INHALATION DEVICE PERFORMANCE}

Lars Karlsson, Mats Josefson, Patrik Andersson, and Staffan Folestad

\section{Pharmaceutical Development, AstraZeneca R\&D Mölndal,} S-43183 Mölndal, Sweden

In this paper an approach is presented where acoustics and multivariate data analysis were applied to pharmaceutical inhalation device characterization. A feasibility study was performed where a set-up of two miniaturized condenser microphones and an analog-to-digital (A/ D) converter was used to monitor the sound of inhalation devices being analysed in either a manual or in an automated delivered dose analysis station. A rig was used to incorporate the Acoustic Emission (AE) system, offering flexibility regarding the placement of the microphones. The raw sound data was Fourier transformed to frequency spectra, which were fed into the multivariate analysis software. It was shown that this is a powerful technique capable of e.g., distinguishing between filled and empty cavities, and between powder mixtures with different properties in the inhalation device, giving an indication on the type and amount of formulation that is used. Valuable metadata (e.g. detecting small differences in pressure drop) related to the quality of the analytical process could also be acquired. Acoustic Emission (AE) can be used to detect differences and provide valuable data on several levels, e.g. between 2 batches of inhalers or between 2 inhalers from the same batch exhibiting minor differences in physical properties that may affect the inspiratory sequence. Early exploration results on $\mathrm{AE}$ as a design aid in the development of a new prototype dry powder inhaler (DPI), is presented here. Special attention is paid to what possibilities various chemometric approaches can offer in terms of data handling of the information rich acoustic signal.

\section{COMBINING PARTICLE ENGINEERING WITH DEVICE DEVELOPMENT TO FINE TUNE DPI PERFORMANCE OF AP301}

Isabel S. Lopes ${ }^{2}$, Bernhard Fisher ${ }^{1}$, Hendrik Fisher ${ }^{1}$, João V. Fernandes ${ }^{2}$, Pedro Serôdio ${ }^{2}$, and Filipe Neves ${ }^{2}$

${ }^{1}$ APEPTICO Forschung und Entwicklung GmbH, Mariahilferstr 136 Top 2.08, A-1150 Vienna, Austria

${ }^{2}$ Hovione FarmaCiencia, SA, Sete Casas,2674-506 Loures, Portugal
AP301 is a new synthetic peptide designed for the treatment of pulmonary lung oedema through the activation of the ENaC channel of type II alveolar cells of the lungs [1]. This product was already administered clinically in the form of a nebulized version, with very promising results in the treatment of patients suffering from Acute Respiratory Distress Syndrome and with primary graft dysfunction following lung transplantation [1]. In order to further expand the range of applications for this product, and given that nebulization is not the preferred delivery route for the vast majority of at home patients, a dry powder inhalation platform was attempted for this product.

For that purpose a spray drying (SD) process was developed in order to produce different Drug Alone powders targeting different Particle Size Distributions (PSD). The objective was to manipulate this property of the powder in order to reduce cohesive / adhesive forces and thus to increase the aerodynamic performance. Throughout the program it was demonstrated that the SD process enabled obtaining stable materials, within the targeted PSD and with a high degree of purity and bioactivity.

Due to the rescue nature of the treatment in some of the intended applications, and due to the difficulty of handling capsule-based devices in emergency situations, the need for patients to have a highly portable and easy to use disposable device was identified. For this reason, a device development program was conducted, targeting the development of an enhanced version of the currently marketed single dose disposable device TwinCaps ${ }^{\circledR}$, able to accommodate large payloads of spray dried powders $[3,4]$. Prototypes of this device and one off the shelf device were used to determine the aerodynamic performance (APSD) of the produced powders. The results indicated the feasibility of obtaining high emitted and fine particle dosages for drug alone powders of AP301, with both types of devices, and that this performance remained stable throughout a 3 month period at different storage conditions $\left(25^{\circ} \mathrm{C} / 60 \% \mathrm{RH}\right.$ and $\left.40^{\circ} \mathrm{C} / 75 \% \mathrm{RH}\right)$.

\section{EVALUATION OF INHALED DRY POWDER TOBRAMYCIN FREE BASE IN NON-CYSTIC FIBROSIS BRONCHIECTASIS PATIENTS}

Marcel Hoppentocht ${ }^{1}$, Onno W. Akkerman ${ }^{2}$, Paul Hagedoorn ${ }^{1}$, Jan-Willem C. Alffenaar ${ }^{3}$, Tjip S. van der Werf ${ }^{2,4}$, Huib A.M. Kerstjens ${ }^{2}$, Henderik W. Frijlink ${ }^{1}$, and Anne H. de Boer ${ }^{1}$

${ }^{1}$ University of Groningen, Department of Pharmaceutical Technology and Biopharmacy, Antonius Deusinglaan 1, 9713 AV Groningen, The Netherlands

${ }^{2}$ University of Groningen, University Medical Center Groningen, Department of Pulmonary Diseases and Tuberculosis, Hanzeplein 1, 9700 RB Groningen, The Netherlands

${ }^{3}$ University of Groningen, University Medical Center Groningen, Department of Clinical Pharmacy and Pharmacology, Hanzeplein 1, 9700 RB Groningen, The Netherlands

${ }^{4}$ University of Groningen, University Medical Center Groningen, Department of Internal Medicine, Hanzeplein 1, 9700 RB Groningen, The Netherlands

\section{Summary}

Bronchiectasis is a persistent condition characterised by dilated and thick-walled bronchi. The presence of Pseudomonas aeruginosa in bronchiectasis is associated with a higher hospitalisation frequency and a reduced quality of life, requiring frequent and adequate treatment with antibiotics.

To assess local tolerability and the pharmacokinetic parameters of inhaled excipient free dry powder tobramycin as free base administered with the Cyclops dry powder inhaler (DPI) to patients with noncystic fibrosis bronchiectasis. The free base and absence of excipients reduce the inhaled powder dose. 
Eight participants in the study were trained in handling the device and inhaling correctly. During drug administration the inspiratory flow profile was recorded. Local tolerability was assessed by spirometry and recording adverse events. Serum samples were collected before and 15, 30, 45, 60, 75, 90, 105, $120 \mathrm{~min} ; 4,8$ and $12 \mathrm{~h}$ after inhalation respectively.

Dry powder tobramycin base was well tolerated and mild tobramycin-related cough was reported only once. A good drug dose serum concentration correlation was obtained. Relatively small inhaled volumes were computed from the recorded flow curves, resulting in presumably substantial deposition in the central airways i.e., at the main site of infection.

In this first study of inhaled dry powder tobramycin free base in non-cystic fibrosis bronchiectasis patients, the free base of tobramycin and the administration with the Cyclops DPI were well tolerated and appreciated. Our data invite further clinical studies to evaluate safety and efficacy of this compound in this patient population.

\section{CHEMICAL-SELECTIVE IMAGING OF ADHESIVE MIXTURES FOR INHALATION USING COHERENT ANTI-STOKES RAMAN SCATTERING (CARS) MICROSCOPY}

Floris Grasmeijer ${ }^{1}$, Andrew L. Fussell ${ }^{2}$, Henderik W. Frijlink ${ }^{1}$, Anne H. de Boer ${ }^{1}$, and Herman L. Offerhaus ${ }^{2}$

${ }^{1}$ Department of Pharmaceutical Technology and Biopharmacy,
University of Groningen, Groningen, The Netherlands
${ }^{2}$ Optical Sciences group, University of Twente, Enschede, The Netherlands

The spatial distribution and degree of agglomeration of drug particles throughout adhesive mixtures for inhalation are thought to be relevant to the dispersion behaviour of such formulations. The physical state of these properties is difficult to assess with conventional high resolution imaging techniques, because the small drug particles often cannot be sufficiently distinguished from other fine mixture components. Chemical-selective imaging techniques may be very helpful in this regard. Although several such techniques have been described, chemical-selective imaging is not yet routinely used in the study of adhesive mixtures. Reasons may be found in insufficient contrast formation for the specific chemicals used in adhesive mixtures for inhalation, or insufficient accessibility of the techniques. Additional techniques are therefore desirable. Here we present a first study on the suitability of coherent anti-Stokes Raman scattering (CARS) microscopy for the chemical-selective imaging of adhesive mixtures. Its combination with high resolution scanning electron microscopy (SEM) is presented. The results show that CARS microscopy is a promising technique for the characterisation of adhesive mixtures. It provides a way for their rapid chemical-selective imaging, even when the drug (e.g. budesonide) does not differ from lactose with respect to the chemical elements of which it is composed. However, the attainable field of view with the CARS setup from this study is limited and not all drug particles appear to be detected with the settings used. Therefore, a further optimisation and validation of the techniques is required.

\section{IN VITRO RESPIRABLE PERFORMANCE OF AMIKACIN DRY POWDER VERSUS AMIKACIN NEBULIZATION}

$\underline{\text { Anna Giulia Balducci }}^{1,2}{ }^{\text {, Fabio Borella }}{ }^{1}$, Ruggero Bettini ${ }^{3}$, Paolo Colombo ${ }^{3}$, and Francesca Buttini ${ }^{3}$

\author{
${ }^{1}$ PlumeStars s.r.l., via Lago Scuro 11, Parma, Italy \\ ${ }^{2}$ Biopharmanet-TEC, Interdepartmental Center, University of Parma, \\ Parco Area delle Scienze 27/A, Parma, Italy \\ ${ }^{3}$ Department of Pharmacy, University of Parma, Parco Area delle \\ Scienze 27/A, Parma, Italy
}

Cystic Fibrosis (CF) is a rare disease affecting 70000 people worldwide. CF patients are particularly susceptible to pulmonary infections caused mainly by bacterial pathogens such as Pseudomonas aeruginosa (PA). To manage chronic exacerbation, antibiotic inhalation is required. The gold treatment is represented by inhalation of tobramycin ${ }^{2}$. However, other aminoglycosides are objects of investigation $^{3}$. The aim of this work was to study in vitro the aerodynamic behaviour of an amikacin sulphate liquid solution after nebulization and an amikacin dry powder (AMK/NaST) after aerosilization from a Dry Powder Inhaler (DPI). AMK/NaST was prepared using a proprietary spray drying technology to construct a powder to inhale having high content of drug and respirability. AMK/NaST powder was manufactured by spray drying and characterized in term of drug loading (\%), morphology and particle size distribution. Then, the aerodynamic assessment of an amikacin sulphate liquid and powder was investigated. Amikacin solution for injection was nebulized with Pari Turbo boy equipped with a Pari LC plus cup. AMK/NaST powder was loaded in the DPI device (RS01, Plastiape) at the labelled dose of $125 \mathrm{mg}$ of amikacin sulphate. The fine particle dose as amikacin base of amikacin solution and AMK/NaST powder was similar $46.4 \mathrm{mg}$ and $47.87 \mathrm{mg}$, respectively. The data indicate that $125 \mathrm{mg}$ of amikacin sulphate powder afford a respirable dose equivalent, or even slightly higher than that obtained by nebulizing $3 \mathrm{~mL}$ of solution for injection containing $625 \mathrm{mg}$ of amikacin sulphate.

\section{BRONCHIAL CHALLENGE TESTING: DIFFERENT HORSES FOR DIFFERENT COURSES}

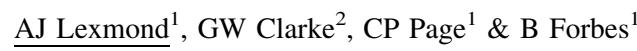

${ }^{1}$ King's College London, Institute of Pharmaceutical Science, 150 Stamford Street, London, SE1 9NH, UK

${ }^{2}$ Quintiles Drug Research Unit, 6 Newcomen Street, London, SE1 1YR, UK

Bronchial challenge tests (inhaled delivery of provocation agents) are performed to measure bronchial hyperresponsiveness (BHR), a characteristic feature of asthma. Measuring BHR is useful in the diagnosis and assessment of asthma, both in a clinical setting and in the development of new asthma therapeutics. For the latter application, bronchial challenge testing has proven its value in studying active and variable airway inflammation and how treatment can alter this. The applications of bronchial challenge tests are diverse, but critically depend on selection of the challenge agent that best suits the study objective and on ensuring that this agent reaches its site of action in the airways reliably and reproducibly.

Despite the numerous challenges that have been performed over the last decennia, surprisingly little attention has been afforded to quantifying the delivery of challenge agents to the airways. The large variations in chemical composition of these challenges (sulphur, methacholine, adenosine, mannitol) and their dose requirements do not allow for a singular approach, which is what has mostly been used to date. Moreover, research groups tend to use their own, non-standardised challenge protocols, impeding comparison of study results across different groups. The diagnostic potential of bronchial challenge tests could greatly be enhanced by quantifying, optimising and standardising the delivery method for each challenge agent. 
Bronchial challenge testing is a neglected field where it comes to drug delivery; its current limitations, potential and unmet needs warrant urgent consideration. By highlighting new applications, both existent and proposed, a future for bronchial challenge testing can be sketched in which there is an imperative opportunity for drug delivery scientists to contribute.

\section{A SCRUTINY OF SCALE: CONSIDERATIONS FOR THE MANUFACTURE OF ORALLY INHALED DRUG PRODUCTS}

\author{
Jeremy Clarke \\ Director, Respiratory CoE, Manufacturing \\ GSK, Priory Street, Ware, Hertfordshire, SG12 ODJ, \\ United Kingdom
}

\section{Summary}

Traditionally, scale considerations for pharmaceutical manufacturing processes have focussed on scale up of the constituent unit operations and generally have been based on empirical approaches. This paradigm is increasingly challenged by regulatory expectations for improved understanding/robustness of manufacturing processes, which drives a need for development of mechanistic models. Other challenges to the scale-up paradigm include the customisation of healthcare through personalised medicines which will demand manufacture of smaller, more frequent, 'on demand' product batches and technological innovations such as continuous manufacturing, where scale-up may either be irrelevant or more modest compared to changes in equipment scale within batch processes.

Specific to Orally Inhaled Drug Products (OIDPs), the lack of a 'SUPAC-like' guidance to inform on required in vitro/in vivo studies to underwrite changes in manufacturing process scale and, in particular, the absence of a simple bioequivalence measure, drives a product/process 'design freeze' earlier in development than for immediate release solid oral dosage forms, which results in the need to develop the commercial scale manufacturing process ahead of pivotal clinical studies. Additionally as a combination product, considerations of scale must be applied to manufacturing processes for both formulation and inhalation delivery device.

In this paper, scale considerations will be reviewed for typical unit operations that comprise the manufacturing process train for a unit dose inhalation powder, identifying the process variables that impact quality of output product, highlighting key best practices and, where possible, where scale-independent mechanistic models can be used to inform technology selection, technology transfer and scale up.

\section{INVESTIGATION INTO THE IN VITRO PERFORMANCE OF ANTI-STATIC VHCS BY PARTICLE SIZE DISTRIBUTION USING THE NEXT GENERATION PHARMACEUTICAL IMPACTOR (NGI)}

$\underline{\text { Scott Courtney and Benjamin Pratt }}$

Medical Developments Int., Research \& Development Dept., 7/56 Smith Rd, Springvale, Melbourne, Australia

\section{Summary}

Aerodynamic particle size distribution and total delivered dose (TDD) performance testing of several Anti-static Valved Holding Chambers (VHCs) has been conducted with a series of selected pMDI drugs using the Next Generation Impactor (NGI) at a flow rate of $30 \mathrm{~L} /$ min. The main body of the Antistatic VHCs tested is made from three distinctively different types of materials of construction-including aluminium (VORTEX Non Electrostatic Holding Chamber), Acrylonitrile Butadiene Styrene, ABS (OptiChamber Diamond Anti-static chamber) and a Polypropylene Blend Polymer (Medical Developments Internationals Anti-static Compact Space Chamber Plus \& AeroChamber Plus Flow-Vu Anti-static VHC). Results have shown that the VHCs made from the polypropylene blend polymer outperform the other VHCs tested for overall TDD and FPD/EFPD deliveries over all drugs tested, with total performance ranking over all three platforms being AS-CSCP > ACP-FV > VOR-NE > OCD-AS.

\section{THE ROLLER COASTER THAT IS AEROSOLIZED BIOMOLECULES. WHAT'S THE NEXT WILD RIDE?}

\author{
Ralph Niven
}

Novartis Pharmaceuticals, 150 Industrial Road, San Carlos, CA 94070 U.S.A.

Inhalation development of biomolecules is typically beset with challenges, as evidenced by the lengthy development of inhaled insulin, and often will not be the first choice as a route of administration. To be successful, the eventual product should be well-differentiated from alternative approaches to therapy and there should be a compelling business case for development. The cost of goods of most biologics is high and can present a significant barrier to early development. Preclinical toxicology drug-substance requirements often exceed mid-stage clinical demand, thus requiring significant early, atrisk investments. Despite the challenges, the inhaled route remains a viable choice for all lung conditions and for selected systemic conditions. This is supported by the availability of highly efficient delivery systems and the fact that the majority of lung diseases are either not well managed with current therapies or have no effective treatment at all. Inhaled vaccines are credible prophylactic options for lung-specific or systemic conditions, while strong cases can be made for the use of select peptides and proteins for systemic disease where a therapeutic advantage can be argued. Although there are few novel clinical-stage biologics currently under development, considerable feasibility and preclinical work is ongoing based on patent and literature activity. What the next 'success' will be is hard to predict but after factoring in attrition rate, logic dictates that many inhaled biotherapeutics will eventually be in clinical use.

\section{AN APPROACH TO PROCESS DEVELOPMENT OF PMDIS USING COLD FILL AND PRESSURE FILL TECHNOLOGY}

\author{
Steve Haswell
}

\section{$3 M$ Drug Delivery Systems, Loughborough, LE11 1EP, UK}

Ideally, projects can be developed quickly and inexpensively, meet customer preferences without impact on the expectation of a single cycle review, are easy to routinely manufacture and batch release and tick all of the boxes that allow a product to be well marketed and available to patients at a competitive price to the health care provider.

To achieve these aims, it is important that the product development and process development scientists work synergistically to develop a thorough understanding of what is required to realize a successful commercial product. Holistic technical and business decisions throughout the development cycle facilitate a smooth transition through product and process development, scale-up, commercial manufacture and launch.

Recent guideline changes and the introduction of Quality by Design $(\mathrm{QbD})$ initiatives further emphasise the requirement to understand the 
relationship between input raw materials, formulation, process design and product performance. These factors, risk assessed and investigated appropriately, together, result in a validated commercial manufacturing process that is both robust and flexible.

The role of the process developer is to facilitate communication along all the touchpoints of the development to commercial spectrum, and to ensure that the right data are being generated at every stage, ensuring that the optimal process, either cold fill or pressure fill is chosen for commercial manufacture.. This paper outlines many of the technical factors that need to be considered during these stages of development, gives some detail behind both manufacturing techniques and discusses the points to consider when choosing cold fill and pressure fill equipment.

\section{FORMULATION AND EVALUATION OF FLUTICASONE PROPIONATE MICROEMULSIONS FOR NEBULISER DEVICES}

\author{
$\underline{\text { Precious Akhuemokhan }^{1}, \text { Mark Parry }^{2} \text {, Mireille Hassoun }}{ }^{1}$, \\ Mark Hammond ${ }^{2}$, and Ben Forbes ${ }^{1}$ \\ ${ }^{I}$ Department of Pharmacy, King's College London, London, \\ $W C 2 R 2 L S, U K$ \\ ${ }^{2}$ Intertek Melbourn, Saxon Way, Cambridge, SG8 6DN, UK
}

\section{Summary}

Purpose: To formulate fluticasone propionate (FP) microemulsions for pulmonary delivery using jet or vibrating mesh nebulisers, characterise the MEs by determining their physicochemical properties and compare their performance against the licensed FP nebuliser suspension (Flixotide ${ }^{\circledR}$ nebules).

Method: The formulations were based on microemulsion formulations licensed for non-pulmonary routes of administration. Three microemulsions (ME1, ME2 and ME3) were prepared and their viscosity and density were measured. The aerosolisation performance of microemulsions and Flixotide ${ }^{\circledR}$ was assessed by measuring delivery rate and respirable dose according to the European pharmacopoeia guidelines. Aerosol particle size distribution was measured by laser diffraction and the aerodynamic size profile was determined by fractionation using a next generation impactor (NGI).

Results: The mesh nebuliser failed to aerosolise any of the microemulsions, whereas the jet nebuliser was effective at delivering ME1, ME2 and Flixotide. The microemulsions had varying viscosities (ME1; 2.64 cP, ME2; 7.74 cP, ME3; 104.00 cP). ME1 and Flixotide had similar delivery rates, but delivered different doses (ME1 27.07\%; Flixotide $52.48 \%$ ). ME2 exhibited the lowest delivery rate and delivered dose. In terms of particle size and aerodynamic particle size distribution, ME2 had the lowest volume diameter for $90 \%$ of droplet population $\left(\mathrm{Dv}_{(90)}\right)$, highest fine particle dose (FPD) and lowest mean mass aerodynamic diameter (MMAD). For ME1, ME2 and Flixotide, respectively, $\operatorname{Dv}_{(90)}=8.71 \mu \mathrm{m}, 7.09 \mu \mathrm{m}, 8.51 \mu \mathrm{m}, \quad \mathrm{FPD}=73.23 \%$, $79.55 \%, 73.96 \%$ and $\mathrm{MMAD}=4.60 \mu \mathrm{m}, 4.18 \mu \mathrm{m}, 4.95 \mu \mathrm{m}$.

Conclusion: The microemulsions in this study showed no major improvement over Flixotide. However, with its higher FPD and lower $\mathrm{Dv}_{(90)}$ \& MMAD values, ME2 is a promising prototype for the formulation of FP microemulsions for jet nebulisers.

\section{MEASUREMENT OF THE PHYSICO-CHEMICAL PROPERTIES OF PHARMACEUTICAL AEROSOLS AS THEY TRAVEL FROM PRESSURISED METERED DOSE INHALERS (PMDI) TO A MODEL LUNG}

\author{
N Davidson ${ }^{1 *}$, H-J Tong ${ }^{2 *}$, M Kalberer $^{2}$, P C Seville $^{3}$, \\ A Ward ${ }^{4}$, M Kuimova ${ }^{5}$ and F D Pope ${ }^{1 *}$
}

${ }^{1}$ School of Geography, Earth and Environmental Sciences, University of Birmingham, Edgbaston, Birmingham, B15 2TT, UK

${ }^{2}$ Department of Chemistry, University of Cambridge, Lensfield Road, Cambridge, CB2 1EW, UK

${ }^{3}$ School of Pharmacy and Biomedical Sciences, University of Central Lancashire, Preston, Lancs, PR1 2HE, UK

${ }^{4}$ Central Laser Facility, Rutherford Appleton Laboratory, Harwell, Oxford, OX11 OQX, UK

${ }^{5}$ Department of Chemistry, Imperial College London, South Kensington Campus, London SW7 2AZ, UK

*Both authors contributed equally to this paper

\section{Summary}

Four different asthma medications were delivered from pressurised metered dose inhalers (pMDI) to a chamber acting as an artificial lung, where they were suspended in a counter-propagating dual beam optical trap. This setup allowed individual drug particles to be suspended in the air in front of a Raman spectroscope while conditions of relative humidity (RH) were altered to mimic conditions of the open air and inside the lungs. Analysis of the resulting Raman spectra allowed direct observation of the drugs' hygroscopic properties, determining whether the particles collect water at high RH and thus change their aerodynamic diameter, altering their deposition within the respiratory tract.

Two beta- 2 agonists, salbutamol sulfate and salmeterol xinafoate, and two steroids, ciclesonide and fluticasone propionate, were analysed in this manner. At greater than $95 \% \mathrm{RH}$, Salbutamol exhibited peak broadening around the polar functional groups in its Raman spectrum. This was interpreted as an indication that the trapped drug particle was collecting water from the air, and images of the particle collected at low and high RH with a charge coupled device (CCD) camera showed a clear increase in particle diameter. By contrast, the steroid ciclesonide shows no changes in particle size or Raman spectrum as RH is raised to close to $100 \%$. This work demonstrates the effectiveness of optical trapping for capturing pharmaceutical aerosols for analysis in more representative conditions than a cover slip, and the usefulness of Raman spectroscopy in monitoring the effects of changing external conditions such as $\mathrm{RH}$ and temperature.

\section{SURFACE MODIFIED VORICONAZOLE DRY POWDER INHALABLE FORMULATION FOR THE TREATMENT OF INVASIVE PULMONARY ASPERGILLOSIS}

\author{
$\underline{\text { Sumit Arora }}^{1-3}$, Mehra Haghi ${ }^{2,4}$, Paul M Young ${ }^{2}$, Michael Kappl ${ }^{3}$, \\ Daniela Traini ${ }^{2}$, and Sanyog Jain ${ }^{1}$
}

${ }^{1}$ Centre for Pharmaceutical Nanotechnology, Department

of Pharmaceutics, National Institute of Pharmaceutical Education and Research (NIPER), Sector 67, S.A.S. Nagar (Mohali)

Punjab- 160062 INDIA

${ }^{2}$ Respiratory Technology, Woolcock Institute of Medical Research and Discipline of Pharmacology, Sydney Medical School,

The University of Sydney, NSW 2037, Australia

${ }^{3}$ Max Planck Institute for Polymer Research, 55128 Mainz, Germany ${ }^{4}$ School of Pharmacy, Graduate School of Health,

University of Technology, Sydney, NSW 2007, Australia

\section{Summary}

Background: Invasive pulmonary aspergillosis (IPA) is a severe disease in immunocompromised patients with extremely high mortality rate. Voriconazole (VRZ) is a first line treatment drug for IPA, conventionally administered orally or intravenous, resulting in a plethora of drug-drug interactions and off-target toxic effects. In the present research work, we developed and characterised a highly 
dispersible dry powder inhalable formulation of VRZ using leucine as a dispersibility enhancer.

Methods: VRZ and leucine in varying concentrations were dissolved in ethanol-water $(70: 30 \% \mathrm{v} / \mathrm{v})$ and spray dried to yield inhalable dry powders. Powders were characterised in terms of particle size, morphology and aerosol performance using the low resistance RS01 dry powder device with next generation cascade impactor. Storage stability (chemical stability and aerosol performance) of the optimized formulation was evaluated for 3 months. Calu-3 sub bronchial epithelial cell line was used to study cell viability (MTS test). Finally, in vivo pharmacokinetic studies in mice were carried out to determine the lung bioavailability of the optimised formulation.

Results: Dry powder comprising VRZ (80\% w/w) and Leucine $(20 \% \mathrm{w} / \mathrm{w})$ was found to be suitable for inhalation therapy. Powder exhibited a volume median diameter of $2.64 \pm 0.05 \mu \mathrm{m}$ and superior aerosolisation with MMAD of $3.79 \pm 0.02 \mu \mathrm{m}$ and fine particle fraction (\% aerosol $<5 \mu \mathrm{m}$ ) of $60.00 \pm 0.94 \%$. Powder exhibited irregular morphology and demonstrated physico-chemical stability of up to 3 months at room temperature. Formulation was found to be non-cytotoxic to Calu-3 cells. Moreover, lung bioavailability in murine model showed the ability of inhaled formulation to attain higher concentration of VRZ in lungs as compared to intravenous administration.

Conclusion: A highly respirable dry powder VRZ formulation was developed for the treatment of IPA.

\section{INFLUENCE OF MANNITOL CARRIER MORPHOLOGY ON THE DPI PERFORMANCE OF DIFFERENT APIS}

\author{
M Mönckedieck $^{1}$, J Kamplade $^{2}$, P Walzel ${ }^{2}$, N Urbanetz $^{3}$, \\ $\mathrm{H}$ Steckel ${ }^{4}$, and R Scherlie $\beta^{1}$ \\ ${ }^{I}$ Department of Pharmaceutics and Biopharmaceutics, \\ Kiel University, Grasweg 9a, 24118 Kiel, Germany \\ ${ }^{2}$ TU Dortmund, Emil-Figge-Str. 68, 44227 Dortmund, Germany \\ ${ }^{3}$ Daiichi Sankyo Real Estate GmbH, 85276 Pfaffenhofen, Germany \\ ${ }^{4}$ Deva Holding AS, Istanbul, Turkey
}

Dry powder inhalers (DPIs) mostly utilise the well-established system of coarse carrier particles $(>50 \mu \mathrm{m}$ ) with good flow properties and active pharmaceutical ingredients (APIs, $<5 \mu \mathrm{m}$ ), which are attached to the carrier surface to overcome dosing problems of the small cohesive drug particles when used alone. This study was employing spray drying with a special focus on particle size and particle morphology to generate batches with tailored mannitol carrier particles of $50-70 \mu \mathrm{m}$. A design of experiments with the factors drying temperature and rotary speed was applied to obtain a wide set of different carrier particles. A choice of six mannitol batches was selected for the preparation of interactive mixtures with both the hydrophilic model drug salbutamol sulphate (SBS) and the lipophilic model drug budesonide. Aerodynamic characterisation was performed with a Novolizer ${ }^{\circledR}$ by using the Next Generation Pharmaceutical Impactor to obtain the Fine Particle Fraction (FPF). Three different SBS qualities were tested. Particle size was found to have a huge impact on the FPF due earlier impaction of larger particles. Additionally, aerodynamic characterisation detected a strong dependence of carrier morphology and FPF of the hydrophilic SBS, which in turn reveals the possibility to control the FPF by the preparation of tailor-made carrier surfaces. The dry powder inhaler performance of lipophilic budesonide was not affected by morphology, but exhibited noticeably higher FPFs for all chosen mannitol batches compared to SBS leading to the assumption that SBS is adhered tighter due to its possibility to build hydrogen bonds.

\section{PHARMACEUTICAL DEVELOPMENT OF SODIUM HYALURONATE RESPIRABLE DRY POWDERS FOR TARGETED DRUG DELIVERY}

\author{
$\underline{\text { F Martinelli }^{1}, \text { AG Balducci }}{ }^{2}$, A Kumar ${ }^{3}$, B Forbes ${ }^{3}$, \\ R Bettini ${ }^{1,2}$, and F Buttini ${ }^{1}$
}

${ }^{1}$ Department of Pharmacy, University of Parma, Viale Parco Area delle Scienze 27/A, Parma, 43124, Italy

${ }_{2}^{2}$ Interdepartmental Center, Biopharmanet-TEC, University of Parma, Viale Parco Area delle Scienze 27/A, Parma, 43124, Italy

${ }^{3}$ King's College London, Institute of Pharmaceutical Science, London SE1 9NH, UK

Background: CD44 is the major hyaluronic acid binding receptor involved in pathological conditions, including tumour progression and metastasis formation. It has been reported that CD44 is a protein overexpressed in non-small cell lung tumours.

The aim of this work was to produce sodium hyaluronate (HA) dry powders using a particle engineering approach based on spray drying technique in view of an efficacious platform capable to deliver chemotherapeutics to the lungs.

Methods: Different dry powder formulations were obtained by spray-drying (SD) water/ethanol mixtures containing HA and a selected adjuvant. HA dry powders were characterized in terms of morphology and in vitro respirability. Biocompatibility of excipients and formulations was investigated using A459 cell culture and the MTT test.

Results: Pure HA SD powders showed a poor aerodynamic performance. Three surfactants were included in the formulations (stearylamine, cetostearyl alcohol and stearyl alcohol) at three different proportions $(10 \%, 5 \%$ and $1 \%)$. Microparticles of HA had a spherical shape with smooth surface; stearylamine led to wrinkled particles whereas cetostearyl and stearyl alcohol kept a roundish shape and some holes were evident onto the surface. Formulations containing 5\% of stearylamine showed the best aerodynamic performance (emitted dose of $89 \%$ and FPF of $52 \%$ of nominal dose).

MTT results ranked the biocompatibility of the excipients: stearylamine $<$ cetostearyl alcohol $<$ stearyl alcohol.

Conclusions: Dry powders suitable for lung delivery were produced by co-spray-drying hyaluronate and surfactants. These might be employed as a platform to directly target tumour cells with antineoplastic drugs.

\section{PDA AND HIGH SPEED IMAGE ANALYSIS OF HFA/ ETHANOL PMDI AEROSOLS: NEW FINDINGS}

\author{
B Myatt ${ }^{1}$, R Newton ${ }^{1}$, D Lewis ${ }^{2}$, T Church ${ }^{2}$, G Brambilla $^{3}$, \\ G Hargrave $^{1}$, H Versteeg ${ }^{1}$, B Gavtash ${ }^{1}$, and E Long ${ }^{1}$ \\ ${ }^{1}$ Wolfson School of Mechanical and Manufacturing Engineering, \\ Loughborough University, Loughborough, LE11 3TU, \\ United Kingdom \\ ${ }^{2}$ Chiesi Limited, Bath Road Industrial Estate, Chippenham, Wilts, \\ SN14 OAB, United Kingdom \\ ${ }^{3}$ Chiesi Farmaceutici SpA, Via Palermo, 43122 Parma, Italy
}

\section{Summary}

The pressurised metered dose inhaler is one of the most popular devices for producing a respirable cloud of droplets or particles for inhaled therapy of respiratory conditions. The primary atomisation mechanism of a pMDI is relatively poorly understood. This work aims to further develop this fundamental understanding by conducting and analysing phase Doppler anemometry (PDA) and high speed imaging studies. 
We report the findings of a PDA study of aerosol plumes generated by a commercial actuator of HFA134a placebo formulations containing ethanol up to $20 \% \mathrm{w} / \mathrm{w}$. Data for the temporal variation of aerosol velocity and droplet size $25 \mathrm{~mm}$ from the spray orifice exit is presented alongside internal flow images of the expansion chamber obtained from custom made transparent models. With increasing ethanol concentration, the spray plume velocity remained broadly constant, but the number mean diameter increased. Internal flow imaging has revealed marked differences in the flow regimes within the expansion chamber between formulations. Previous studies have suggested that the aerosol velocity and droplet size are strongly linked with the formulation vapour pressure, a function of ethanol concentration. The variation of flow regime with ethanol concentration and mean droplet diameter increase observed in this study suggest that other physical properties (e.g. surface tension and/or viscosity) play more significant roles in governing the drop size than previously acknowledged.

This study demonstrates that increasing the concentration of ethanol in the formulation increases the droplet size produced. However, the ethanol concentration does not have a discernible effect on the aerosol plume velocity.

\section{DOES MUCIN BINDING HAVE AN EFFECT ON THE INHALED DISPOSITION OF ENAC BLOCKERS?}

\author{
Melania Giorgetti ${ }^{1}$, Jesus Angulo ${ }^{1}$, Nichola Smith ${ }^{2}$, Brian Cox $^{3}$ \\ David Sandham ${ }^{2}$, and Chris Morris ${ }^{1}$ \\ ${ }^{1}$ University of East Anglia, Norwich Research Park, \\ Norwich, NR4 7TJ, UK
}

${ }^{2}$ Novartis Institute of BioMedical Research, 100 Technology Square, Cambridge, MA 02139, USA

${ }^{3}$ Department of Chemistry, University of Sussex, Brighton BN1 9QJ, Sussex, UK

\section{Summary}

Mucus is a key component of the pulmonary mucosal defence system trapping inspired microorganisms and particulate material. The main "non-water" component of mucus is the macromolecular glycoprotein, mucin, which is principally responsible for the viscoelastic gel nature of mucus. Mucin forms a complex gel network that presents both polar glycosylated domains and un-glycosylated nonpolar domains that may serve as drug binding pockets. Indeed, evidence suggests that some inhaled drug molecules can be bound by mucus and their diffusion rate significantly retarded. In this work we examined the mucin binding of a panel of inhaled drug candidates with the aim of identifying the key drug physicochemical parameters which predict mucin binding.

We present data for the mucin binding of a panel of epithelial sodium channel $(\mathrm{ENaC})$ blockers, including amiloride and its analogues, which have been studied as experimental therapeutics for cystic fibrosis (CF). Ultrafiltration binding assays were performed to examine the binding characteristics for a panel of analogues which spanned a $\log \mathrm{P}$ and molecular weight range of -0.89 to 1.45 and 230 to 756 , respectively. A strong positive correlation was observed between the extent of binding and drug $\log \mathrm{P}$, whereas parameters such as molecular weight, polar surface area and molecular volume were weakly predictive of mucin binding. Molecular level information about these drug-mucin interactions was obtained from Saturation Transfer Difference-NMR spectroscopy studies. These studies identified "interacting" and "noninteracting" domains within the drug molecule. The study highlights the potential for mucin to impact upon drug disposition in the lung. 